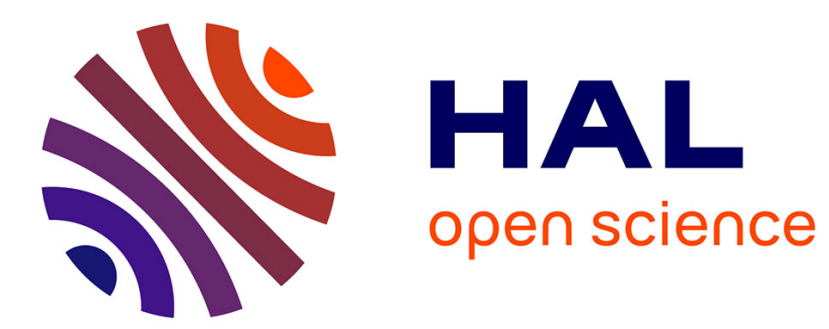

\title{
Sur les apocryphes bouddhiques chinois
}

Liying Kuo

\section{- To cite this version:}

Liying Kuo. Sur les apocryphes bouddhiques chinois. Bulletin de l'Ecole française d'Extrême-Orient, 2000, 87 (2), pp.677-705. 10.3406/befeo.2000.3496 . halshs-02514989

\section{HAL Id: halshs-02514989 \\ https://shs.hal.science/halshs-02514989}

Submitted on 23 Mar 2020

HAL is a multi-disciplinary open access archive for the deposit and dissemination of scientific research documents, whether they are published or not. The documents may come from teaching and research institutions in France or abroad, or from public or private research centers.
L'archive ouverte pluridisciplinaire HAL, est destinée au dépôt et à la diffusion de documents scientifiques de niveau recherche, publiés ou non, émanant des établissements d'enseignement et de recherche français ou étrangers, des laboratoires publics ou privés. 


\section{Sur les apocryphes bouddhiques chinois}

Liying Kuo

\section{Citer ce document / Cite this document :}

Kuo Liying. Sur les apocryphes bouddhiques chinois. In: Bulletin de l'Ecole française d'Extrême-Orient. Tome 87 N², 2000. pp. 677-705;

doi : https://doi.org/10.3406/befeo.2000.3496

https://www.persee.fr/doc/befeo_0336-1519_2000_num_87_2_3496

Fichier pdf généré le 08/02/2019 


\author{
Abstract \\ Kuo Liying \\ On Chinese Buddhist apocrypha
}

"Buddhist apocrypha" is conventionally used by western scholars to translate the words "fake and dubious sutras" used by Chinese bibliographers, i.e. Buddhist texts, mainly sutras, directly written in Chinese in contrast to genuine Buddhist texts, which are necessarily translated from an Indian or Centralasiatic original. Some texts that were at first condemned as fake were nevertheless considered genuine afterwards and were widely read and used by Chinese, Japanese and Korean Buddhists. Other apocrypha, being rejected from the official Canon, usually soon disappeared. Some are nevertheless preserved because of exceptional circumstances: many known apocrypha were discovered from Dunhuang or Nanatsudera in Japan. This paper is concerned with the reasons Chinese bibliographers gave for classing some texts as fake and to be excluded from the Canon and with the use of apocrypha in controversies between Buddhists and Taoists. Trying to improve upon studies of dated Dunhuang apocrypha copies by Fujieda Akira, it deals with the evolution in their contents and appearance between the 5th and 10th centuries and contains a quantitative study of their output. Special consideration is then

given to three kinds of apocrypha, mainly (sometimes only) known from the Dunhuang find, viz. surras whose reading and copying should avoid calamities or procure benefits such as health, merits to be transferred to defuncts etc.; sutra-offerings, i.e. sutras specially copied for being used and offered during religious ceremonies for getting the same benefits as above; and - quite different in their goal and use - esoteric handbooks, tantras and dhārani-sutras, some of which are specific to Dunhuang.

\title{
Résumé
}

Kuo Liying

Sur les apocryphes bouddhiques chinois

Dans la sinologie en langues occidentales, le terme « apocryphe bouddhique » désigne des textes, surtout des sutra, stigmatisés comme faux par les auteurs chinois de catalogues parce que composés directement en chinois alors que les ouvrages dits authentiques sont nécessairement des traductions de textes en langues indiennes ou d'Asie centrale. Quelques ouvrages d'abord considérés comme faux, mais ensuite reclassés parmi les authentiques, ont joué un grand rôle dans le bouddhisme chinois, coréen et japonais. D'autres ouvrages, exclus du canon, ont en général disparu. Seules des circonstances exceptionnelles ont permis leur conservation : beaucoup d'apocryphes sont connus par les trouvailles de Dunhuang et du Nanatsudera au Japon. Le présent article étudie l'évolution de la notion de "faux " chez les bibliographes chinois, son utilisation dans les controverses bouddhotaoïques et son rapport avec la diffusion des canons impériaux. Reprenant et précisant de précédentes études de Fujieda Akira consacrées aux copies datées de Dunhuang, il propose une vue générale de l'évolution du contenu et de l'apparence des apocryphes du Ve au Xe siècle et en donne une étude quantitative. II étudie enfin trois catégories d'apocryphes bien et parfois uniquement représentées à Dunhuang : les sutra dont la lecture et la copie permettent d'éviter des catastrophes, d'obtenir une bonne santé ou des mérites pour les parents décédés, etc. ; les sutra d'offrande utilisés dans des cérémonies destinées à obtenir ces mêmes biens ; et - fort différents - des manuels rituels, sutra de dhârani ou tantra dont certains sont spécifiques à la région de Dunhuang. 


\title{
Sur les apocryphes bouddhiques chinois
}

\author{
KuO Liying
}

\section{Introduction}

Nous devons beaucoup aux savants qui, comme Eugène Burnouf (1801-1852) ou Friedrich Max Müller (1823-1900), ont édité les premiers textes bouddhiques à partir de manuscrits apportés en Europe, sans avoir pu jamais aller sur le terrain ${ }^{1}$. Leur seule connaissance du bouddhisme vivant venait de rares récits de voyageurs qui en général comprenaient mal ce qu'ils voyaient. Max Müller bénéficia aussi de renseignements donnés par des étudiants venus d'Orient. Eux-mêmes étaient particulièrement conscients de ce que leur information, entièrement livresque, était partielle. C'est ainsi que Max Müller encouragea 1'un de ses élèves japonais, Takakusu Junjirō 高楠順次郎 (18661945), à traduire le récit du pèlerin chinois Yijing 義淨 (635-713) sur les règles de vie des moines indiens ${ }^{2}$. C'est sans doute aussi une des raisons pour lesquelles Sylvain Lévi (1863-1935) tint à se rendre au Népal.

La nécessité où les savants occidentaux se trouvèrent malgré eux d'étudier le bouddhisme dans les seuls textes eut deux conséquences. La première fut de rechercher d'abord l'état le plus ancien des textes et des doctrines comme si c'était le meilleur. La deuxième fut de tendre à considérer le bouddhisme comme une "conception du monde ", une philosophie rationaliste, pas une religion. Ce qui revenait à dire que le bouddhisme médiéval et contemporain était une dégénérescence sans intérêt.

Ce sont trois savants formés sur le terrain et tout autant philologues, Alfred Foucher (1865-1952), Paul Demiéville (1894-1979) et Paul Mus (1902-1969), qui contribuèrent le plus à faire bouger les choses. Puis les contacts réguliers avec les bouddhistes japonais, chez eux, et les bouddhistes tibétains exilés rétablirent ce qui est aujourd'hui une vérité admise : le bouddhisme n'est pas seulement une philosophie, un abhidharma, c'est une religion, c'est-à-dire une pratique, des rituels, un mysticisme et des aspects qui varient selon le temps, le lieu, la culture et la personnalité de l'adepte. Il n'y a pas dégénérescence, mais évolution. Cette évolution dépend parfois de la culture et des modes de pensée du milieu où le bouddhisme s'est propagé et n'est pas moins intéressante à étudier - sur place et dans les textes - que le plus lointain passé de la doctrine. On découvre ainsi de nouveaux aspects de la vie intellectuelle et de nouveaux champs d'études s'ouvrent. La mise en place au Japon par Sylvain Lévi, Takakusu Junjirō et Paul Demiéville du Dictionnaire encyclopédique du bouddhisme d'après les sources chinoises et japonaises, Hōbōgirin, résulta de la prise de conscience de la nécessité d'une coopération

1. Burnouf, 1844 et 1852 ; Müller, 1881 ; Müller et Nanjio, 1883. Cf: Yuyama, 2000.

2. Takakusu, 1896. 
internationale permettant de connaître le bouddhisme à travers les textes et les pratiques. Le travail de présentation, de traduction et de commentaire des érudits chinois et japonais, eux-mêmes moines croyants et pratiquants, put ainsi être mis à la disposition des indianistes et des spécialistes du bouddhisme d'autres régions du monde.

La découverte des manuscrits de Dunhuang, à l'aube du $\mathrm{XX}^{\mathfrak{e}}$ siècle, permit un accès direct à des textes anciens et médiévaux, disparus, ou conservés seulement dans des éditions postérieures de beaucoup aux originaux et susceptibles de les avoir modifiés. Ces manuscrits sont loin d'avoir été totalement édités, étudiés et utilisés. Il reste encore beaucoup à faire, notamment dans l'étude des pratiques anciennes. Je prendrai pour exemple le domaine des apocryphes chinois, où la combinaison d'études philologiques, de recherches sur le terrain, de connaissance des mentalités et la coopération internationale sont une nécessité première.

\section{Apocryphe et authentique}

Les sütra transmettant la parole du Buddha, il est important d'en garantir l'authenticité, gage de la vérité de la doctrine. Les Tibétains se sont posé ce problème et l'ont résolu en gardant dans leurs bibliothèques les originaux indiens, preuves de l'authenticité des textes qu'ils avaient traduits, le fait que les textes aient été traduits du sanskrit leur paraissant suffire à démontrer l'authenticité du message qu'ils transmettaient. Confrontés à la multiplication des textes, les bouddhistes indiens eux-mêmes se sont interrogés sur l'authenticité de certains d'entre eux. Les critères varient selon les sectes et les écoles. La polémique a de nombreux échos dans les textes mahāyāniques, comme Étienne Lamotte (1903-1983) l'a bien montré ${ }^{3}$. Plus récemment, Ronald Davidson est revenu sur ce sujet ${ }^{4}$. Le Mahāpadesasutta pali donne une règle qui resta théorique. À un moine qui prétendrait démontrer l'authenticité d'un point doctrinal en se référant à un enseignement reçu directement par lui (1) du Buddha, (2) d'une communauté organisée avec des moines anciens (thera), (3) de thera instruits, (4) d'un unique thera instruit, il faut seulement opposer la lettre et l'esprit des textes (sütra et vinaya). S'il y a accord, le propos est admis ; si non, il est rejeté ${ }^{5}$.

\section{Formule introductive du sūtra}

La formule introductive des sütra, donnant le lieu et l'occasion où le sermon fut prononcé (evam mayā śrutam ekasmin samaye), en garantit l'authenticité. Elle a donné lieu à de nombreux débats sur la ponctuation de la phrase ou la façon de la lire, c'est-àdire de séparer par une pause plus ou moins longue les groupes de mots ${ }^{6}$. Cette formule est généralement traduite en chinois par rushi wowen yishi 如是我聞一時, “c'est ainsi que $\mathrm{j}$ 'ai entendu en une circonstance ", depuis le début du ve siècle, probablement sous l'influence de Kumārajiva qui arriva à Chang'an aux alentours de $402^{7}$. La formule est

3. Lamotte, 1947 et 1949.

4. Davidson, 1990.

5. Lamotte, $1947: 220 ; T .1451,24: 389 \mathrm{~b}$. Cette règle est traduite en chinois comme sida jiaofa 四大教法, 《quatre grands critères par rapport à l'enseignement 》, dans le Chang Ahan jing 長阿含經 (Dìrghāgama), T. 1(3), 1: 17c-18a (les références au Taishō sont données par numéro, volume, page et registre). 1999.

6. Brough, 1950 ; von Hinüber, $1968: 84-87$; Silk, 1989 ; Calloway, 1991 ; Tola et Dragonetti,

7. Zürcher, $1980: 99$. 
expliquée et son usage approuvé dans le troisième chapitre du Traité de la grande vertu de sagesse de Nāgārjuna dont le texte en chinois est dû au même Kumārajiva ${ }^{8}$. Une bonne partie des sūtra censés avoir été traduits durant le $\mathrm{II}^{\mathrm{e}}$ siècle l'omettent. Le sūtra commence directement par fo zai 佛在..., « le Buddha se trouvait à ... ». C'est notamment le cas dans les sütra traduits par Zhiloujiachen 支婁迦纖 (Lokakșema), qui travailla en Chine entre 167 et $186^{9}$. La formule introductive, evam mayā śrutam ekasmin samaye, est régulièrement traduite en chinois à partir $\mathrm{du} \mathrm{II}^{\mathrm{C}}$ siècle seulement, et encore avec de petites variantes, soit dans l'ordre des mots, soit dans le choix de ceux-ci ${ }^{10}$. Ces variations pourraient servir d'indice pour connaître la date de la traduction d'un sūtra et peut-être aussi déterminer si le texte ainsi traduit n'est pas en fait un apocryphe ou une copie d'extraits de sütra" .

\section{" Apocryphes " et sūtra rédigés directement en chinois}

La notion d'apocryphes est une notion chinoise ancienne. Les bibliographes chinois distinguent en effet entre sütra « vrais 》 (zhen 畺), traduits d'une langue indienne, et sütra «faux » (wei 俘), composés directement en chinois. Les savants occidentaux, reprenant une expression utilisée par les biblistes, parlent d'apocryphes et désignent par ce terme les

8. Le Dazhidu-lun 大智度論, T. 1509(3), $25: 66 \mathrm{a}-67 \mathrm{a} ;$ Lamottc, 1944-80, t. I : 80-88.

9. J. Brough (1950: 417) l'avait noté pour la traduction de Zhiloujiachen du Käśyapaparivarta (T. 350). On peut voir que les trois autres traductions de ce sūtra effectuées sous les Jin (265-420), T. 351, sous les Qin (351-431), T. 310 (43e assembléc), et par Shihu 施護 (982-1017), T. 352, incluent toutes une formule introductive. Celle des Jin a la formule du III siècle (wen rushi yishi fo you 聞如是一時佛遊 ... 《Entendu ainsi en une circonstance le Buddha voyageait ... ») et les deux autres ont celle $\mathrm{du} \mathrm{V}^{\mathrm{e}}$ siècle (rushi wowen yishi fo zai 如起我聞--時佛作..., «Ainsi j’ai entendu en une circonstance le Buddha se trouvait à ... »). On peut se demander pour quelle raison les traducteurs du ${ }^{\mathrm{e}} \mathrm{e}^{\mathrm{C}}$ siècle n'ont pas traduit cette formule. Est-il possible que les manuscrits en langues indiennes qu'ils utilisaient ne l'aient pas comportéc ? De même les textes pali et sanskrit des sütra font précéder la formule ekam samayam par une phrase ou une stance d'hommage au Buddha, aux bodhisattva, etc. On peut le constater aisément en ouvrant le Saddharmapuṇdarika, le Sukhāvatìȳiha et les Prājñ̄āpāramitā, etc. Mais ces phrases ne se trouvent presque jamais au début des sūtra traduits en chinois. Il est certain aussi que nous devons mettre en question l'attribution traditionnelle des traductions. Comme le constate E. Zürcher (1991: 278), les auteurs des catalogues sont très généreux dans leurs attributions. À travers les catalogues successifs, le nombre de traductions d'ouvrages attribuées, par exemple, à An Shigao (II ${ }^{\mathrm{C}}$ s.), passe de 34 à 179. Celui des traductions attribuées à Lokakșema passe de 2 à 23. Six des 11 sütra du Canon du Taishō attribués à Lokakṣema n'ont pas la formule introductive. Sur 51 textes attribués à An Shigao par le Taishō, cinq seulement n'ont pas la formule. Vingt textes ont la formule du ve siècle. Pour une augmentation considérable du nombre de traductions attribuées au même auteur à partir des Souei (fin $\mathrm{VI}^{\mathrm{e}}$ et début $\mathrm{VII}^{\mathrm{e}}$ s.), voir Ono Gemmyō, $1936: 28-29$.

10. En gros, la situation est la suivante : 1) $\mathrm{II}^{\mathrm{e}} \mathrm{s}$., pas de formule introductive dans nombre de sütra;

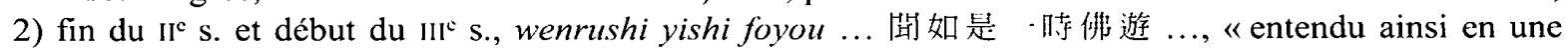
circonstance le Buddha voyageait à...", parfois mais rare : wenrushi fozai...聞如是佛在...., « entendu ainsi le Buddha se trouvait à...»; 3) deuxième moitié du III ${ }^{\mathrm{e}}$ s. au IV $\mathrm{IV}^{\mathrm{e}}$ s., wenrushi yishi fozai... 聞如是-一時佛在 ..., 《entendu ainsi en une circonstance le Buddha se trouvait à... », parfois mais plus rare: wowen rushi yishi fozhu... 我聞如是一..時俳住..., “ $\mathrm{j}$ 'ai entendu ainsi en une circonstance le Buddha demeurait à... »;4) à partir du début $\mathrm{v}^{\mathrm{e}} \mathrm{s}$., rushi wowen yishi fozai... 如是我聞一時佛在..., «c'est ainsi que j'ai entendu en une circonstance le Buddha se trouvait à... ». La quatrième formule devient alors standard, même si parfois fo (Buddha) est remplacé par Boqiefan 溥晸梵, transcription de Bhagavat, ou par Shizun 世尊, «Vénéré du Monde ».

11. Deux sütra, l'un sur les préceptes de bodhisattva, l'autre sur les bodhisattva-bhümi, furent catalogués par Sengyou comme copies et extraits de sütra car ils n'ont pas la formule rushi wowen, T. 2145(9), 55 : 62c-63a. Je remercie Mme Sylvie Hureau de m'avoir indiqué cette référence. 
textes bouddhiques rédigés ou recomposés directement en chinois. Le terme est commode mais ambigu. Il désigne aussi les « faux sūtra » exclus du canon officiel par les auteurs des ouvrages bibliographiques chinois ${ }^{12}$ bien que certains d'entre eux, quoique fabriqués en Chine, aient joué un rôle de premier plan dans le bouddhisme chinois, dans celui du Japon et de la Corée. Ainsi les deux sütra traitant des préceptes de bodhisattva, le Fanwang jing 梵網經 ou Sütra des filets de brahman $(T .1484)^{13}$ et le Pusa yingluo benye jing 菩薩聵珞本業經 ou Sūtra traitant des karma fondamentaux de bodhisattva (T. 1485), le sütra qui indique les méthodes divinatoires pour connaître l'avenir, le Zhancha shan'e yebao jing 占察善惡業報經 ou Sūtra traitant des bonnes et mauvaises rétributions karmiques par le moyen divinatoire $(T .839)^{14}$, le sütra utilisé pour le rituel de protection de l'État, le Renwang poruo poluomi jing 仁王般若波羅密經 ou Sütra de la sagesse des Bons Rois $(T .245,246)^{15}$. On sait aussi que la plupart des textes traduits en chinois, même s'ils l'ont été à partir de textes en langues de l'Inde ou de l'Asie centrale, ne sont pas tous issus de l'enseignement du Buddha. On peut même se demander s'il existe un seul sütra reproduisant directement la parole du Buddha. Pourquoi donc un texte composé au $\mathrm{V}^{\mathrm{e}}$ siècle en Chine serait-il moins authentique qu'un texte composé au Cachemire à la même époque ${ }^{16}$ ?

Pour l'étude du bouddhisme chinois, la question de l'authenticité de la parole n'est pas l'essentiel. Ce qui est intéressant, c'est de voir la façon dont on a procédé pour emprunter et adapter en Chine une pratique et une philosophie religieuses provenant d'un pays dont les langues, les écritures et les modes de penser sont profondément différents. L'authenticité de la parole ou du texte indien traduit était en fait impossible à vérifier pour un Chinois bouddhiste du $\mathrm{V}^{\mathrm{e}}$ siècle. Il lui fallait faire confiance à celui qui l'avait apporté ou traduit. Par ailleurs ces bouddhistes chinois tenaient à pratiquer la religion bouddhique comme dans son pays d'origine ${ }^{17}$. Tout ce qui venait du pays du Buddha était précieux et sacré. On voulait donc inclure dans le canon chinois un maximum de textes. C'est la raison pour laquelle les moines chinois et coréens ont risqué leur vie et sont partis en Inde recueillir les enseignements du Buddha et les textes sacrés utilisés dans les communautés bouddhiques indiennes afin de les rapporter fidèlement à leurs compatriotes. L'admiration des moines chinois pour les maîtres ou choses venant de l'Inde se voit aussi dans les Biographies des moines éminents (Gaoseng zhuan 高僧傳, T. 2059, 2060, 2061, etc.).

\section{Fabrications des faux chez les Chinois}

Les auteurs chinois de catalogues d'ouvrages bouddhiques condamnent sévèrement les textes qui n'ont pas été traduits directement d'une langue non chinoise ou sont de provenance inconnue. Ils les classent comme "erronés 》 (wang 安), "superficiels » (wei 僞) ou encore "faux et douteux qui troublent les textes authentiques 》 (yiwei luanzhen 疑鹞亂㑑). Leur mission, souligne Zhisheng 智昇 (669-740), est de faire que «les enseignements vrais soient dans la bonne voie et que la parole d'or [du Buddha] aboutisse à une œuvre ${ }^{18}$.

12. Buswell, $1990: 3-5$.

13. Le sūtra est traduit en français par De Groot, 1893. Pour les sūtra des préceptes de bodhisattva: Demićville, 1930. Cf. également Groner, 1990.

14. Lai, 1990 et Kuo, 1994b.

15. De Visser, 1935, vol. I : 116-142 ; Orzech, 1998.

16. Strickmann, $1982: 57$ et Seidel, $1990: 289$.

17. On se souvient que le grand maître des vinaya, Dao'an 道安 (314-385), avait souffert de ne pas savoir avec certitude comment cette religion était d'origine. Cf. Kuo, 1994a : 26.

18. T. 2154(1), $55: 477 \mathrm{a}$; Tokuno, $1990: 32$. 
Sengyou 僧祐 (445-518), vers 515, relève quelques cas typiques de fabrication de $s \bar{u} t r a$, dont certains de son temps. La production de faux sütra est souvent considérée comme le résultat d'une révélation divine (shenshou 神授) ou d'une connaissance acquise dans une vie antérieure (suxi 宿習). C'est le cas de la nonne Sengfa 僧法 (fin du $\mathrm{V}^{\mathrm{e}} \mathrm{s}$., début du $\mathrm{VI}^{\mathrm{c}} \mathrm{s}$.) et d'une dame nommée Ding 丁氏 (fin du $\mathrm{II}^{\mathrm{c}} \mathrm{s}$.). La dame Ding aurait prononcé les sütra en une langue des Hu (胡語) sans jamais l'avoir apprise. Un religieux nommé Miaoguang 邚; 光 contemporain de Sengyou, à Nankin, au $V^{\mathrm{e}}{ }^{\mathrm{s}}$ siècle, s'était spécialisé dans la fabrication de faux sūtra pour en tirer profit. Il avait fabriqué un sūtra intitulé Saporuotuo juanshu zhuangyan jing 薩婆若陀春屬㽝嚴經 ou Sūtra de la famille ornée de Saporuotuo. Sengyou note que Saporuotuo est le nom du père de Miaoguang. Dans le $s \bar{u} t r a$, le nom de son frère est Jingangdeti 金涺德體 (Corps de la vertu du vajra), et celui de son disciple est Shizi 師 子 (Fils du maître ou Lion). Miaoguang avait établi un culte autour de son sūtra et s'était attiré la confiance de fidèles féminines qu'il grugeait à son aise. La communauté dont Sengyou faisait partie finit par le livrer à la justice séculière. Il fut emprisonné à Dongye 東治, dans la province de Fujian, loin de Nankin. Une vingtaine de copies de ce « faux sūtra » fut brûlée devant la préfecture ${ }^{19}$.

Les souverains chinois, en effet, ont toujours voulu contrôler la religion de leur peuple. Dès le début de la transmission du bouddhisme en Chine, l'entreprise de traduction des sütra fut étroitement contrôlée par la cour impériale. Les grands catalogues d'ouvrages bouddhiques furent compilés à la demande de l'Empereur. La protection de l'État était une nécessité pour les bouddhistes chinois qui, du $\mathrm{V}^{\mathrm{c}}$ au $\mathrm{IX}^{\mathrm{c}}$ siècle, connurent trois fois la persécution, en 446,574 et 844 , sous les règnes des trois empereurs Taiwu des Wei (424-451), Wu des Zhou (561-577) et Wuzong des Tang (841-846).

Les bouddhistes durent aussi faire face à la concurrence taoïste. Dans ce pays qui respecte tant l'écriture, pour faire face aux flots de textes bouddhiques traduits, composés et rédigés en Chine, les taoïstes composèrent leur propre canon en empruntant beaucoup aux textes bouddhiques. Ils rédigeaient parfois des textes montrant que le bouddhisme est la reproduction des enseignements de leur grand maître Laozi. Le Laozi huahu jing 老子化胡經 ou Sütra de la conversion des Barbares par Laozi prétend ainsi que Laozi serait parti à l'Ouest et s'y serait incarné en Buddha Śākyamuni. Les bouddhistes ne restèrent pas insensibles à ces attaques. Ils fabriquèrent entre autres le Qingjing faxing jing 清浄法行經 ou Sūtra de la pratique de pureté, qui fait de Laozi, Confucius et Yan Hui 顏回, le disciple le plus célèbre de Confucius, les incarnations de trois disciples du Buddha envoyés par leur maître en Chine pour civiliser le pays. Le Daode jing 道德經 ou Livre de la voie et de la vertu (attribué à Laozi) et le Xiao jing 孝經 ou Livre de la piété filiale (attribué à Confucius) seraient ainsi les enseignements du Buddha ${ }^{20}$. Les bouddhistes accusent aussi les textes taoïstes de n'être que des imitations et modifications des sütra bouddhiques. Ils les qualifient de "faux classiques» (weidian 傜典), "classiques hérétiques » (xiedian 邪典), parfois aussi 《faux sūtra» (weijing 傜經), la même expression qui est utilisée pour les sūtra bouddhiques fabriqués en Chine. Ils les dénoncent à l'autorité impériale ${ }^{21}$. Ils n'hésitent pas à utiliser ces faux sütra taoïques pour montrer la supériorité des textes bouddhiques ${ }^{22}$. Ils préviennent les taoïstes du danger qu'il y a à faire des faux. Ils citent le cas du Sanhuang jing 三皇經 ou Sūtra des Trois

19. T. 2145, 55 : 40b-c ; Demiéville, 1954-55: 248 ; Strickmann, $1990: 100-102$.

20. Zürcher, $1959: 288-320$.

21. T. 2103,52 : 150c-151b et 174b-c ; T. $2110,52: 543 b-546 b$ (Bokenkamp, $1983: 467-468$ ); voir aussi $T .2056,49: 581 \mathrm{~b}-\mathrm{c}$.

22. T. $2103,52: 162 \mathrm{a}-\mathrm{c}$. 
Empereurs, du $\mathrm{V}^{\mathfrak{c}}$ siècle, brûlé sur ordre de la cour impériale ${ }^{23}$. Ils racontent le cas d'un taoïste du début du $\mathrm{VII}^{\mathrm{e}}$ siècle, nommé Huixiang 慧祥, qui, après trois ans sans prononcer un seul mot, aurait fabriqué, en prenant le modèle du Nirvāna-sūtra, un texte appelé Chang 'an jing 長安經 ou Sūtra de la longue paix. Celui-ci reçut d'abord l'ordre de ne pas quitter la ville, puis, accusé d'avoir porté des vêtements d'une couleur impériale, fut exécuté à la porte de la ville ${ }^{24}$.

\section{Signification des apocryphes chinois}

Parmi les textes bouddhiques classés comme "faux », certains semblent en fait des traductions peu fidèles ou de libres adaptations. Ils sont faux en tant que textes, mais la doctrine est orthodoxe. La tradition scientifique occidentale les dit "apocryphes », mot ainsi devenu synonyme du "faux» des auteurs chinois. Nous suivons cette tradition. Toutefois il faut signaler que les grands maîtres chinois, parfois auteurs de catalogues, n'ont pas hésité à donner comme preuves de vérité des citations provenant des sūtra classés comme faux par eux-mêmes. Ils écrivirent également des commentaires sur ces " faux sütra» comme ils le faisaient pour les grands sütra authentiques, tels le Sütra du Lotus et les Mahāprājñāpāramitā-sütra. Il est certain que les sūtra composés ou fabriqués en Chine conviennent mieux au public chinois. Les apocryphes bouddhiques, pour la plupart, mêlent des notions chinoises aux croyances et pratiques indiennes afin de mieux faire adopter celles-ci par les Chinois. Les auteurs d'apocryphes utilisent les expressions religieuses taoïstes et morales des confucéens. Ils introduisent des croyances populaires chinoises. L'étude de ces "faux » permet donc de mieux comprendre la façon dont le bouddhisme chinois, coréen et japonais s'est constitué et a évolué.

\section{Critères chinois d'authenticité}

Les critères d'authenticité du bouddhisme indien (si da jiaofa 四大教法) sont repris par Sengyou dans son introduction à la section " apocryphes » de son catalogue, compilé vers 515. Sengyou cite également une phrase du Mahāparinirvāna-sūtra prédisant qu'après le nirvāna du Buddha, des bhikșu copieront et fabriqueront des sūtra et classiques (chaozao jingdian 抄造經典) entraînant l'affaiblissement de la Loi [du Buddha] ${ }^{25}$. De plus Sengyou se plaint que la fabrication de faux sütra ait augmenté depuis l'époque de la Loi contrefaite (xiangfa 像法 / pratirūpaka-dharma). Mais Sengyou est seul à évoquer la possibilité de fabrication des faux textes par des bouddhistes indiens en Inde même. À notre connaissance, aucun autre auteur chinois ne porte de doute sur les textes en langues indiennes ou d'Asie centrale. Les catalogues, compilés en 593 (T. 2146), en $602(T .2147)$ et en $730(T .2154)$, se contentent de réunir dans une section particulière les textes intitulés souvent jing ( $s \bar{u} t r a$ ) dits être traduits du sanskrit, mais rédigés après la mort du Buddha par ses disciples (shengxian jizhuan 㘹賢集傳 ou shengxian zhuanji lu 聖賢傳記錄 $)^{26}$. Zhisheng 智开 dit que ces textes ne sont pas des classiques proprement dits (zhengdian 正典) des Trois Corbeilles (Sanzang 三藏/Tripitaka), mais ils aident à

23. T. 2103(8), 52 : 14lb16-19. Le Sanhwang jing aurait été remplacé par le Daode jing de Laozi vers la fin de l'ère Zhenguan (627-649), T. 2122(65), 53 : 708a-b. Mais il existait encore à l'époque des Song (Chen Guofu, $1963: 76-78$ ).

24. T. $2104,52: 141 \mathrm{a}$ et $386 \mathrm{~b} ;$ T. $2110(8), 52: 549 \mathrm{a} ; T .2051,50: 209 \mathrm{~b} ;$ T. $2116(1), 52: 756 \mathrm{a}$. Pour les emprunts de textes bouddhiques dans les écrits taoïstes : Zürcher, 1980 et Bokenkamp, 1983 et 1990.

25. T. 2145(5), $55: 38 \mathrm{c}$. La citation du Nirvāna-sūtra se trouve à $T .374,12: 421 \mathrm{c}-422 \mathrm{a}$.

26. T. 2146(6), $55: 144 \mathrm{a}-148 \mathrm{~b} ;$ T. $2147(2), 55: 161 \mathrm{~b}-\mathrm{c} ; T .2154(13), 55: 621 \mathrm{c}-625 \mathrm{~b}$. 
propager le bouddhisme. Pour cette raison les ouvrages rédigés par les saints personnages, les textes bibliographiques, biographiques, monographiques, les traités et manuels rituels composés et rédigés par les moines chinois, méritent d'être inclus dans le Canon à côté des paroles du Buddha. Il ajoute que les textes biographiques traduits du sanskrit doivent être rangés avant ceux qui ont été rédigés en Chine ${ }^{27}$.

En Chine, le critère d'authenticité ne concerne que les textes écrits en chinois, et se résume à la question de savoir s'ils ont été réellement traduits d'une langue non chinoise, indienne ou de l'Asie centrale. Il vaut surtout pour les sütra, mais il existe aussi quelques textes de vinaya et de śástra considérés faux. Le plus connu est le Dachen qixin lun 大乘起信論 ou Traité de la production de la foi selon le Grand Véhicule, objet d'un bon travail de Paul Demiéville ${ }^{28}$.

Les entreprises de traduction étaient très contrôlées par la bureaucratie. Le nouveau sūtra devait être présenté à la cour impériale avant d'être diffusé. Il n'est pas rare qu'un empereur ou un haut fonctionnaire séculier ait rédigé une préface ou un éloge pour un sūtra fraîchement traduit. Les auteurs des ouvrages bibliographiques reproduisent ces préfaces et éloges. Ils notent les noms du traducteur et aussi parfois la date. Ils réservent d'ailleurs une section pour les textes dont les noms de traducteurs sont inconnus (shiyi 失譯), parfois traités comme des apocryphes.

Un texte peut être considéré comme faux également pour des raisons de style ou de contenu. Les sütra authentiques sont dits être écrits en un style beau et élégant tandis que les faux sūtra sont souvent écrits d'une façon grossière et vulgaire (wenjie qianbi 文偈淺鄙) et contiennent un sens et des doctrines contradictoires (yili guaibei 義理耕背) ${ }^{29}$. Si un nom du traducteur est donné pour un sūtra soupçonné d'être faux, on le compare avec un autre sütra du même traducteur pour voir si les deux textes ont le même style. Pour les bibliographes chinois, les faux sütra sont des fabrications issues de «complots des hommes» (renmo 人謨) mises frauduleusement sous le nom du Buddha ; ce sont des fabrications par des hommes (renzao 人作), pas des paroles du Buddha (foyu 佛語 / buddhavācana) ${ }^{30}$.

La condamnation d'un texte comme faux ne se faisait pas à la légère. Quand il s'agit d'un catalogue compilé sur ordre impérial, le bureau chargé de sa rédaction était composé d'environ soixante-dix moines dont six chargés de vérifier l'authenticité des sütra (kanding zhenweijing seng 刊定眞傜經僧). Ainsi en est-il du catalogue rédigé sur ordre de l'impératrice Wu des Tang en 695 (T.2153). Déjà le catalogue rédigé par Fajing 法經 et ses confrères sur ordre de l'empereur en 594 séparait pour la première fois les textes considérés comme "douteux et troublants» (yihuo 疑惑) de ceux jugés être "faux et erronés » (weiwang 倸安) ${ }^{31}$. Zhisheng, qui termine en 730 un catalogue compilé de sa propre initiative, réexamine ceux qui étaient qualifiés de douteux (yihuo zaixiang 疑惑再詳) ${ }^{32}$.

Les auteurs des bibliographies classent à part les copies de sütra (chaojing 抄, 經) qui sont infidèles au texte originel ou qui sont des assemblages d'extraits. À propos de deux textes qui sont des extraits doctrinaux copiés par un moine nommé Fayuan 法願, Sengyou

27. T. $2154(13), 55: 621 \mathrm{c}$.

28. Demiéville, 1929. Le traité est qualifié de «śăstra douteux et troublant» (yihuo) dans le catalogue de Fajing, rédigé en 594 ( $T$. 2146, 55 : 142a16-18).

29. T. $2145(5), 55: 38 \mathrm{c} 28$ et $39 \mathrm{a} 20$. Cf. Tokuno, $1990: 36-37$.

30. T. $2153(15), 55: 747 \mathrm{c} 11-13$.

31. T. $2146(7), 55: 148 \mathrm{~b}-\mathrm{c}$.

32. T. 2154(18), 55: 671b-680a. Mais certains sütra énumérés dans la liste des douteux à réexaminer se trouvent également dans celle des «sütra faux et erronés». 
précise que ces copies sont une façon de répandre la doctrine du Buddha. Ce ne sont pas des textes fabriqués (yiyu weizao 異於嗳造), mais ils peuvent passer à l'avenir pour des ouvrages originaux. Afin d'éviter cette confusion, Sengyou décida de les inclure dans la section des textes jugés être faux ${ }^{33}$.

\section{Étude quantitative}

Voici le nombre de textes classés comme apocryphes dans quelques catalogues édités dans le Canon de Taisho ${ }^{34}$. Je donne aussi le pourcentage par rapport à l'ensemble des ouvrages indiqués dans le catalogue dans les cas où cela est possible.

1. Le catalogue rédigé par Dao'an en 374 est perdu. Selon Sengyou, qui l'a utilisé, il qualifiait 26 ouvrages (30 rouleaux) de «sütra douteux », 142 ouvrages (147 rouleaux) de textes « nouvellement recueillis dont on ignore le nom du traducteur » (shiyi) ${ }^{35}$. On ignore le nombre de traductions authentiques. Celles-ci ont été intégrées par Sengyou dans son catalogue.

2. En 515, le catalogue de Sengyou contient 419 titres d'ouvrages authentiques traduits en chinois ${ }^{36}$. Aux 26 ouvrages douteux de Dao'an, Sengyou ajoute 20 ouvrages en 26 rouleaux (soit en tout 46 ouvrages en 56 rouleaux) ${ }^{37}$. Aux 142 ouvrages en 147 rouleaux sans nom de traducteur, il ajoute 460 ouvrages en 675 rouleaux (soit en tout 602 ouvrages en 822 rouleaux) ${ }^{38}$.

Sur un total de $419+46+602=1067$ titres, 419 soit $39,27 \%$ sont authentiques, 46 soit $4,31 \%$ sont douteux et 602 soit $56,41 \%$ sont sans garantie d'authenticité.

3. Le catalogue de Li Kuo 李廓, rédigé entre 531 et 551 et perdu, citait selon Fei Changfang 費常房 (597) 62 ouvrages considérés comme «faux sütra» (fei zhen jing 非葚經), 4 《faux śástra 》(fei zhenlun 非真論) et 11 ouvrages 《totalement faux (quanfei jing 全非經), que [seuls] des hommes stupides pouvaient prendre pour des (vrais) sūtra ${ }^{39}$.

4. Le catalogue de Fashang 法上, rédigé entre 570 et 576 et perdu, citait selon Fei Changfang 51 ouvrages (106 rouleaux) «fabriqués par les hommes » (ruzuo lu 人作錄) ${ }^{40}$.

5. Le catalogue de Fajing, rédigé en 593 sur ordre de l'empereur, fournit des listes chiffrées, soit :

- 784 sūtra mahāyāniques (1 718 rouleaux) dont 21 (30 rouleaux) «douteux et troublants » et 80 (196 rouleaux) « faux et erronés »;

- 842 sütra hīnayāniques (1 031 rouleaux) dont 29 (31 rouleaux) «douteux et troublants », et 53 (93 rouleaux) « faux et erronés »;

- 50 ouvrages (82 rouleaux) de vinaya mahāyānique (sic) dont 1 (2 rouleaux) " douteux et troublant » et 2 ( 11 rouleaux) « faux et erronés »;

- 63 ouvrages (381 rouleaux) de vinaya hīnayānique dont 2 (3 rouleaux) « douteux et troublants » et 3 ( 3 rouleaux) "faux et erronés »;

- 68 ouvrages ( 281 rouleaux) d'abhidharma mahāyānique dont 1 (1 rouleau) « douteux et troublant » et 1 ( 1 rouleau) « faux et erroné »;

- 116 ouvrages (482 rouleaux) d'abhidharma hīnayānique : 1 (1 rouleau) « douteux et troublant » et 2 ( 10 rouleaux $)$ "faux et erronés ${ }^{41}$.

33. T. 2145(5), $55: 39 \mathrm{~b} 4-7$.

34. Voir aussi Makita, $1976: 25-27$.

35. T. $2145(5), 55: 38 \mathrm{~b}-\mathrm{c}$ et T. $2145(3), 55: 16 \mathrm{c} 7-18 \mathrm{c} 2$.

36. T. $2145(2), 55: 5 \mathrm{c} 5-6$.

37. T. $2145(5), 55: 39 \mathrm{~b} 13-15$.

38. T. 2145(4), $55: 21 \mathrm{~b}-37 \mathrm{~b}$.

39. T. 2034(15), 49: 126a9-21. Ce catalogue est appelé parfois «Catalogue de Damoyuduoluo (Dharma-uttara) » ou « Catalogue des Qi [rédigé sous le règne de la famille] Gao », cf. Kuo, 1999 : 524-525. 40. T. 2034(15), $49: 126 \mathrm{a} 23-\mathrm{b} 4$. 
On voit que les faux sont particulièrement nombreux dans la catégorie des sütra, ellemême la plus nombreuse. Au total, sur 1923 ouvrages recensés, 142 soit 7,38\% sont déclarés « faux » et 55 soit $2,86 \%$ sont « douteux ».

6. Le catalogue de Yancong 彦琮, rédigé en 602, cite 2109 ouvrages (5058 rouleaux) dont 209 (490 rouleaux) «douteux et faux » $(9,91 \%)^{42}$.

7. Le catalogue de Daoxuan 道宣, rédigé en 644, ajoute aux 26 ouvrages douteux de Dao'an, aux 20 ouvrages douteux de Sengyou, aux 31 ouvrages douteux de Fei Changfang, aux 28 ouvrages douteux de Fajing 25 titres d'ouvrages douteux et 20 titres de copies de sütra ${ }^{43}$. Sur un total de 2232 titres $^{44}, 20$ seraient ainsi des doubles (des copies). Resteraient donc 2212 titres de traductions dont $26+20+31+28+25=140$, soit $6,33 \%$, déclarées douteuses.

8. Le catalogue des ouvrages conservés au monastère Dajing'ai 大敬愛寺 de la capitale des Tang, compilé par Jingtai 靜泰 sur ordre de l'empereur en 666, cite 29 ouvrages douteux ( 31 rouleaux) et 53 faux (93 rouleaux) ${ }^{45}$.

9. Le catalogue compilé en 695 par Mingquan 明詮 sur ordre de l'impératrice Wu des Tang donnait 3616 titres (8641 rouleaux) dont 228 (419 rouleaux) de «faux $»{ }^{46}$, soit $6,30 \%$. Sur les 228 ouvrages faux, 22 (29 rouleaux) étaient des textes de la secte des Trois Degrés (Sanjie jiao 二階教) ${ }^{47}$.

10. Le catalogue de Zhisheng 智昇 ou catalogue de l'ère Kaiyuan, compilé en 730, donne 2278 titres d'ouvrages ( 7046 rouleaux $)^{48}$ dont 14 (19 rouleaux) douteux à réexaminer, et 392 ( 1055 rouleaux) déclarés faux ${ }^{49}$, en tout 406 ouvrages $(14+392)$, soit $17,82 \%$.

11. Le catalogue de Yuanzhao 圓照 dit catalogue de l'ère Zhenyuan, compilé en 800 , donne 2447 titres d'ouvrages (7399 rouleaux) ${ }^{50}$ dont 14 (19 rouleaux) douteux mais à réexaminer et 393 (1 491 rouleaux) déclarés faux ${ }^{51}$. Le nombre des authentiques a donc augmenté en 70 ans de 169 ouvrages, celui des faux de 1 seulement ${ }^{52}$.

Une récapitulation donne les chiffres suivants :

\begin{tabular}{l|l|l}
\hline année & nombre de faux ou douteux & pourcentage sur l'ensemble \\
\hline 374 & $26(+142)$ & $1 /$ \\
\hline 515 & $46(+460)$ & $4,31 \%(+56,41 \%)$ \\
\hline 593 & $142+55$ & $7,38 \%+2,86 \%=10,24 \%$ \\
\hline 602 & 209 & $9,91 \%$ \\
\hline 644 & 140 & $6,33 \%$ \\
\hline 695 & 228 & $6,30 \%$ \\
\hline 730 & 406 & $17,82 \%$ \\
\hline 800 & 407 & $16,63 \%$ \\
\hline
\end{tabular}

41. T. $2146(7), 55: 148 \mathrm{c}-150 \mathrm{a}$.

42. T. $2147,55: 150 \mathrm{~b}$.

43. T. $2149(10), 55: 333 \mathrm{c}-336 \mathrm{a}$.

44. T. 2149(1), $55: 219 \mathrm{c} 13-15$.

45. T. 2148(4), $55: 211 \mathrm{c} 18-212 \mathrm{c} 22$.

46. T. $2153(1), 55: 371 \mathrm{a} 7-9$.

47. T. 2153(15), $55: 472 \mathrm{a} 22-475 \mathrm{a} 15$. Un décret de 695 ordonna de classer les textes de cette secte parmi les apocryphes. En 699, toutes les pratiques ascétiques et religieuses de la secte furent interdites (p. 475a). Sur cette secte, voir Yabuki, 1927 et Lewis, 1990.

48. T. $2154(1), 55: 477 \mathrm{a} 22-26$.

49. T. $2154(18), 55: 671 \mathrm{~b} 19$ et $672 \mathrm{a} 8$.

50. T. $2157(1), 55: 771 \mathrm{~b} 6-7$.

51. T. $2157(1), 55: 909 c 20-21$.

52. Makita Tairyō (1976:34) pense que le chiffre de 393 pourrait être erroné, car, dit-il, il devait y avoir beaucoup plus de faux en 800 qu'en 730 . On notera aussi la disproportion du nombre des rouleaux, beaucoup plus important en 800 qu'en 730 . 
Ces chiffres méritent commentaire. Les bibliographes n'ont pas enregistré tous les textes dits bouddhiques qui circulaient parmi les fidèles dans tout l'empire chinois. De certains textes, on ne connaît que le titre. Ainsi les textes qui auraient été prononcés par la jeune nonne Sengfa ont été inclus dans presque tous les catalogues. Or il semble que personne ne les ait jamais vus, pas même Sengyou son contemporain. Des textes classés comme vrais ont pu par la suite être déclarés faux ou vice versa. Par exemple, des apocryphes anciens ( $\mathrm{IV}^{\mathrm{e}}-\mathrm{VI}^{\mathrm{e}} \mathrm{s}$.), le Piluo sanmei jing 䍡羅二.昧經 ou Sūtra du samādhi Piluo, le Guanshiyin sanmei jing 觀世音三.昧經 ou Sūtra du samādhi d'Avalokiteśvara, le Qingjing faxing jing 清淨法行經 (mentionné plus haut), le Zuimiao shengding jing 最妙勝定經 ou Sūtra de la concentration suprême, et le Jueding zuifu jing 決定罪福經 ou Sütra qui définit le mérite et le péché, etc., en tout huit sūtra, sont donnés comme authentiques par le catalogue de l'impératrice Wu en 695. Trente-cinq ans plus tard, Zhisheng les déclasse et les range parmi les textes «douteux et troublants à réexaminer $"{ }^{53}$. On remarque également que les textes dont on ne connaît plus le nom du traducteur sont nombreux. De l'époque de Dao'an à celle de Sengyou, leur nombre est multiplié par quatre.

Le pourcentage d'apocryphes augmente brutalement au virI siècle : il double. Mais la quantité des apocryphes suit une progression différente. De 515 à 593 , le nombre de titres est multiplié par 4 environ. De 602 à 730 , il double encore. On peut donc dater des années 500-700 la grande période de production des apocryphes.

\section{Apocryphes et Canon officiel}

Les auteurs des ouvrages bibliographiques disent vouloir classer les ouvrages en « authentiques » et «faux » pour que les authentiques soient conservés et les apocryphes rejetés (cun zhengjing, qu weiben 仔正經, 去䳕本) ${ }^{54}$. Les apocryphes sont donc exclus du Canon ou des bibliothèques officielles. Ils ne doivent plus circuler ou même être utilisés par les fidèles. Daoxuan suggère de ne pas recopier les apocryphes, les copies d'extraits et autres compilations (biesheng 別生). Quand il s'agit d'un texte existant en un seul exemplaire (danben 單本), d'une nouvelle traduction (zaifan 再翻), d'un recueil de biographies de saints personnages (shengxian jizhuan 聖賢集傳), il propose de l'inclure dans le Canon (ruzang 入藏) ${ }^{55}$. En 658, le canon du monastère Ximing 西明寺 de la capitale, donc la liste des livres dont la copie était autorisée, comprenait 799 livres (3 361 rouleaux $)^{56}$. Moins d'un siècle plus tard, en 744, le canon de Zhisheng compte 1076 ouvrages (50 048 rouleaux répartis en 480 enveloppes). La liste de Zhisheng (ruzang lu 入藏錄) ${ }^{57}$ devient la liste officielle des textes canoniques censés avoir été traduits avant l'an $730^{58}$. En 794, un autre moine du monastère Ximing, Yuanzhao 圓照, y ajoute des sütra, notamment tantriques, traduits depuis 730 (T. 2156). L'importance de ces listes officielles tient au fait qu'elles sont approuvées par l'autorité impériale et que celle-ci, en principe, les fait respecter dans toute l'étendue de l'empire. Les sūtra déclarés apocryphes

53. T. 2154(18), $55: 671 \mathrm{~b}-\mathrm{c}$.

54. T. $2153,55: 372 \mathrm{c}-373 \mathrm{a}$.

55. T. $2149(10), 55: 337 \mathrm{c} 9-16$.

56. T. $2149(10), 55: 337 \mathrm{c} 17-20$.

57. T. 2154(19, 20), 55: 680a-700c [reproduction de l'édition coréenne de 1151], voir aussi p. 700c-723a [reproduction de l'édition des Ming (1601) comparée avec celles des Song (1239) et des Yuan (1290)].

58. Hayashiya, 1941 : 15-16, 103-109 ; Fang, 1991. À titre indicatif, le Canon de Taishō comprend 2184 ouvrages pour la section chinoise et en tout, avec les rédactions japonaises, 3053 ouvrages en 17970 rouleaux. 
et exclus de ce canon bouddhique ont peu de chances de subsister. Lors de la composition ou recomposition d'une bibliothèque, ils ne seront pas recopiés. Les exemplaires anciens disparaîtront par usure. Les apocryphes auraient donc dû totalement disparaître de la circulation. Certains pourtant ont pu continuer à exister, ceux par exemple qu'un bibliographe reconnaissait comme authentiques ou à réexaminer, même si d'autres bibliographes les jugeaient faux: les catalogues ne s'accordent pas toujours entre eux. C'est le cas du Zhancha shan'e yebao jing, du Fanwang jing, etc. D'autres textes ont été partiellement conservés sous forme d'extraits cités dans les ouvrages encyclopédiques et dans les commentaires de sütra et les traités doctrinaux. C'est le cas du Qingjing faxing jing, du Jingdu sanmei jing 泎度二昧經 ou Sūtra du samādhi [qui conduit au] pur salut, du Tiwei Poli jing 提謂波利經 ou Sütra de Trapusa et Bhallika ${ }^{59}$, etc.

Il ne faut pas oublier que la composition des fonds de bibliothèques des monastères variait. À lire Daoxuan, il semble que les copies d'apocryphes aient été plus nombreuses dans les collections des monastères non impériaux et de particuliers (renjian jingzang 人間經藏) que dans celles des monastères impériaux ${ }^{60}$. Les fonds de bibliothèque en outre ne provenaient pas seulement d'acquisitions financées par l'État. Il y avait des dons en argent d'origine locale et des dépôts d'ouvrages copiés ${ }^{61}$. Les entreprises de copie n'étaient pas seulement exécutées sur ordre impérial. Les autres membres de la famille impériale ou les hauts fonctionnaires entreprenaient également cet acte de dévotion. Le don du livre se développe beaucoup chez les aristocrates chinois des Six Dynasties et des Tang, qui ne se limitaient pas à faire copier les grands sütra. Ils faisaient également copier et parfois copiaient eux-mêmes d'autres types de sūtra, notamment ceux relatant des miracles relatifs aux besoins de l'existence humaine tels que les conçoivent les Chinois : avoir des descendants, guérir des maladies, prolonger la vie, assurer le destin après la mort de parents décédés, etc. Les fidèles moins fortunés ou même pauvres pratiquaient également ce type d'acte méritoire, espérant ainsi obtenir une vie meilleure à plus ou moins court terme. C'est à ces actes méritoires de particuliers que nous devons l'existence d'une soixantaine de sütra apocryphes et textes de la secte des Trois Degrés à Dunhuang, et d'une autre vingtaine de sütra apocryphes du $\mathrm{XII}^{\mathrm{e}}$ siècle dans un monastère de la banlieue de Nagoya, Nanatsudera ${ }^{62}$.

\section{Manuscrits de Dunhuang et apocryphes}

En 1911, peu de temps après sa visite à la grotte des manuscrits de Dunhuang, Paul Pelliot avait prononcé un discours devant l'Académie des inscriptions et belles-lettres pour signaler l'importance des apocryphes bouddhiques en Chine et en Asie centrale ${ }^{63}$. Il fut le premier à attirer l'attention du monde occidental sur ces textes, exceptionnellement conservés parmi les manuscrits de Dunhuang, que les spécialistes du bouddhisme ne songeaient pas à étudier. En fait, il fallut attendre 1954 pour qu'un autre savant occidental, Paul Demiéville, reprenne ce thème et en fasse le sujet de cours au Collège de France ${ }^{64}$.

59. Pour le Jindu sanmei jing, voir Makita, 1971 ; pour le Sütra de Trapuṣa et Bhallika, voir Makita, $1976: 148-211$ et Lai, 1987.

60. T. 2149(10), $55: 336 \mathrm{al}$ 16-17.

61. À propos de la constitution des fonds de bibliothèques de monastères, voir Drège, 1991 : 194-208.

62. Ochiai, 1991 et 1996.

63. Pelliot, 1911.

64. Demiéville, 1954-1955. 
Les savants japonais l'avaient précédé en ce domaine ${ }^{65}$ et P. Demiéville savait sa dette envers eux.

\section{Chronologie des apocryphes de Dunhuang}

Fujieda Akira, l'un des grands spécialistes des manuscrits de Dunhuang, s'était beaucoup intéressé aux problèmes de chronologie. Il y a à Dunhuang environ 1000 manuscrits datés (toutes catégories confondues). Le plus ancien remonte à l'an 406 et le plus récent à 1002. Ces manuscrits datés permettent d'établir une échelle paléographique. Appliquée aux apocryphes dont le colophon comporte une date et à ceux dont le colophon n'en comporte pas, elle permet de constater que les apocryphes ont été copiés aux Ve et VI siècles et aux $I^{e}$ et $X^{c}$ siècles. Presque rien aux VIIre et VIII ${ }^{e}$ siècles. D'après lui, les apocryphes copiés aux $\mathrm{V}^{\mathrm{e}}$ et $\mathrm{VI}^{\mathrm{e}}$ siècles sont des sütra alors considérés comme authentiques et qui furent plus tard exclus des catalogues impériaux, mais apparemment conservés dans une (des ?) bibliothèque(s) de monastère ordinaire. Ainsi le Datong fangguang jing 大通方廣經 ou Sūtra large et étendu de la grande perspicacité ${ }^{66}$, le Jingdu sanmei jing ou Sūtra du samādhi [qui conduit au] pur salut, Tiwei Poli jing ou Sütra de Trapuṣa et Bhallika ${ }^{67}$ et les Foming jing 佛名經 ou Sütra des noms de buddha et les textes de la secte des Trois Degrés. Les copies des $\mathrm{IX}^{\mathrm{c}}$ et $\mathrm{X}^{\mathrm{c}}$ siècles sont très différentes, de moins bonne qualité, et apparemment pas destinées à une bibliothèque de monastère ${ }^{68}$.

Il est clair qu'il y a une différence de nature et de contenu entre les apocryphes fabriqués aux $V^{*}$ et $V^{c}$ siècles et ceux fabriqués aux $I^{*}$ et $X^{c}$ siècles. Les apocryphes fabriqués aux $V^{e}$ et $V^{e}$ siècles cherchent à se faire passer pour des sütra traduits des langues indiennes. Ils reproduisent la structure des sūtra indiens, et leur formule introductive, evam mayà śrutam ..., etc. Les plus caractéristiques (Jingdu sanmei jing et Tiwei Poli jing) ont été rédigés en 446 , quelques années après la fin de la persécution du bouddhisme par l'empereur Wu des Wei du Nord et la restauration du bouddhisme. Bien qu'exclus du canon officiel, ils n'avaient rien de véritablement hétérodoxe et ont continué à être lus et à exister dans certaines bibliothèques de monastères notamment provinciaux et même parfois dans les collections privées. Rappelons que Daoxuan disait que les textes apocryphes se trouvaient plus facilement dans les collections non impériales que dans les bibliothèques officielles (ci-dessus p. 687). Certains monastères étaient plus laxistes que d'autres, même appartenant à la même région. Makita Tairyō a ainsi constaté que le catalogue d'un monastère de Dunhuang dont le nom nous est inconnu ne contient aucun texte apocryphe (S. 2142), alors que deux catalogues du monastère Sanjie 二界寺 (S. 3624 et 6225) de Dunhuang mentionnent des apocryphes. Il remarque également que les deux listes du IX $\mathrm{X}^{\mathrm{c}}$ siècle, étudiées par Fujieda, ne mentionnent aucun apocryphe. Il suggère, probablement avec raison, que ces deux listes servaient à déterminer les sütra à copier afin de constituer des canons conformes à celui du catalogue de Zhisheng en 730 . Il constate aussi avec étonnement que, dans S. 2079, deux apocryphes du $\mathrm{IV}^{\mathrm{e}}$ siècle, le Jingfu jing

65. Ainsi Yabuki Keiki (1927), Ono Genmyō (1936), Hayashiya Tomojirō (1941), Mochizuki Shinkō (1946), et plus récement Makita Tairyō (1976).

66. À propos de ce sūtra et de son rite, voir Makita, $1976: 290-303$ et Kuo, $1994: 139-140$. Un manuscrit de Dunhuang de la collection de Matsumoto Bunsaburō 松本文一. 郎 a servi de base à l'édition de $T .2871,85: 1338 \mathrm{c}-1355$.

67. Les manuscrits P. 3732, S. 2051 et shuang 霜 15 de Pékin ont été édités par Makita, 1976 : 184-209.

68. Fujieda, $1973: 121$ et 124. 
敬福經 ou Sütra traitant les respects des [actes] méritoires ${ }^{69}$ et le Zhancha [shan'e yebao] jing (mentionné ci-dessus), sont inclus dans une liste « des sütra dits nouvellement traduits 》 (xin fanjing mulu 新翻經日錄) ${ }^{70}$. La date exacte de S. 2079 est inconnue, mais les ouvrages énumérés sont ceux conservés au monastère Longxing 龍興寺 durant l'époque tibétaine, c'est-à-dire au moins deux siècles après la composition de deux apocryphes ${ }^{71}$. Fang Guangchan signale la mention des apocryphes dans deux autres manuscrits, P. 3202 et P. 4962, contenant également des listes d'ouvrages de ce même monastère, ainsi le Shouluo biqiu jing 首羅比斥經 ou Sūtra du bhikșu Shouluo, un apocryphe du $\mathrm{VI}^{\mathrm{c}}$ siècle $^{72}$. Fang constate ainsi que malgré Daoxuan et Zhisheng, chaque monastère à Dunhuang gardait une certaine liberté dans le choix des textes qu'il conservait ou considérait comme canoniques. Les apocryphes repérés n'étaient pas classés à part mais mêlés aux sūtra et ouvrages canoniques. J.-P. Drège confirme les indications de ces catalogues : les apocryphes dont le texte a été conservé seulement parmi les manuscrits de Dunhuang y sont mêlés à d'autres textes de toutes sortes, sütra ou même catalogues d'ouvrages bouddhiques. Ils pouvaient être rangés avec eux dans un même emballage ou sur une même étagère ${ }^{73}$. Mais la variation par rapport aux catalogues officiels est minime.

\section{Manuscrits du Nanatsu-dera}

En 1990 fut identifiée, dans la bibliothèque du Nanatsu-dera, une série de manuscrits $\mathrm{du} \mathrm{XII}^{\mathrm{e}}$ siècle. Une vingtaine étaient des copies d'apocryphes chinois. Il y avait également quelques sūtra fabriqués au Japon. L'édition de ces textes vient d'être achevée sous la direction de MM. Makita Tairyō et Ochiai Toshinori ${ }^{74}$. Ce fonds contient l'unique exemplaire du texte complet d'un apocryphe célèbre, souvent cité dans le débat entre bouddhistes et taoïstes pendant les Six Dynasties, le Jingqing faxing jing ${ }^{75}$. Le Jingdu sanmei jing, qui traite en partie des péchés et des enfers, et qui semble issu d'un milieu bouddhiste et taoïste à la fois, y est conservé en version longue, en trois rouleaux ${ }^{76}$. On y a retrouvé aussi la version du Foming jing en seize rouleaux qui est probablement la base de toutes les autres versions apocryphes du même sütra ${ }^{77}$. La seule version aujourd'hui

69. Le sūtra est mentionné pour la première fois dans le catalogue de 593, T. 2148(4), $55: 212$ b9. Le titre mentionné par Makita est une abréviation. Le manuscrit indique en fait : Rulai zai jin 'guan zhulei

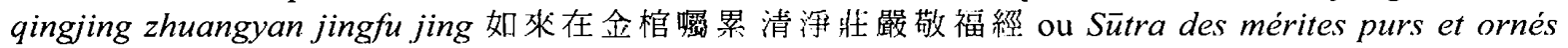
de la "mise en dépôt" (parindanā) par le Tathāgata dans son cercueil d'or (titre traduit par Durt, 1996: 2, n. 2). Un fragment de ce sütra se trouve dans le manuscrit S. 208 et est édité dans T. 2877, $85: 1361 \mathrm{bl-13.}$

70. Makita, $1976: 36-38$. On remarquera aussi que le catalogue de Daoxuan, Neidian lu 內 典錄, est également inclus dans cette liste.

71. Le manuscrit S. 2079 est en fait incomplet. Fang Guangchang (1991: 356-414) a reconstruit une liste plus complète de la collection de ce monastère à l'aide de P. 3807 .

72. Fang, 1991 : 144-145. Le sūtra est mentionné pour la première fois par Fajing en 593 comme apocryphe sous le titre de Shouluo biqiu jian Yueguang tongzi jing 首羅比丘見 几光童子經, Sūtra du bhikșu Shouluo qui rencontre le garçon Yueguang (Candraprabha kumāra) (T. 2147(4), 55: 173bl9). Le texte est conservé dans le manuscrit S. 2697 et édité dans T. 2873, 85: 1356a-1358c. Le début manque. Cf. Yabuki Keiki, 1933 : 220-226; Zürcher, 1982.

73. Drège, $1991: 215$.

74. Makita et Ochiai, 1994-2000. Voir également Kuo, 1995b et 1999.

75. Le texte est édité, traduit en japonais et étudié dans Makita et Ochiai, 1994-2000, vol. $2: 5-28$ et 745-782. Une traduction annotée en français a été faite par le groupe de recherches sur les apocryphes (UMR 8583 du CNRS et EFEO). Elle sera publiée prochainement dans un volume thématique sur les apocryphes en l'honneur du professeur Makita Tairyō. Cf. ci-dessus, p. 681.

76. Makita et Ochiai, 1994-2000, vol. 2 : 29-349 et 926-904.

77. Makita et Ochiai, 1994-2000, vol. 3 ; Kuo, 1995a. 
connue du Piluo sanmei jing 毘羅三昧經 ou Sütra du samādhi Piluo ${ }^{78}$, perdu depuis fort bien longtemps en Chine, s'y trouve aussi. Ce sütra aurait été classé par Dao'an en 374 comme « douteux ». Avec d'autres sütra (Qingjing faxing jing et Guanshiyin sanmei jing, etc.), il avait été déclaré authentique par le catalogue de l'impératrice Wu des Tang en 695 et finalement rejeté comme faux par Zhisheng en 730 (supra, p. 686). Le Guanshiyin sanmei jing, connu par les écrits des deux grands maîtres chinois Zhiyi 智顗 (538-597) et Jizang 吉藏 (549-623), se retrouve à Dunhuang et existe également au Nanatsu-dera, avec quelques variantes par rapport aux copies de Dunhuang ${ }^{79}$.

\section{Apocryphes et cultes populaires à Dunhuang}

Les copies datées d'apocryphes des $\mathrm{VII}^{\mathrm{e}}, \mathrm{VIII}^{\mathrm{e}}, \mathrm{IX}^{\mathrm{e}}$ et $\mathrm{X}^{\mathrm{e}}$ siècles, notait Fujieda Akira, n'ont pas été faites par des copistes professionnels. Elles n'étaient pas destinées aux bibliothèques. Le contenu des textes ainsi copiés est un mélange de doctrine bouddhique et de croyances « vulgaires » (« a mixture of Buddhism and vulgar faiths ») ${ }^{80}$.

Ces conclusions, exactes dans leur ensemble, doivent pourtant être nuancées. Certains apocryphes des $\mathrm{V}^{\mathrm{e}}$ et $\mathrm{VI}^{\mathrm{e}}$ siècles sont ainsi recopiés aux $\mathrm{IX}^{\mathrm{e}}$ et $\mathrm{X}^{\mathrm{e}}$ siècles quand ils servaient au culte. Ainsi le Sütra des noms de buddha dont les copies datent de 596 et de $920^{81}$. Des apocryphes du $\mathrm{VII}^{\mathrm{e}}$ siècle, le Fumu enzhong jing 父母恩重經 ou Sütra concernant les bienfaits des deux parents, le Foshuo xuming jing 佛說續命經 ou Sütra pour continuer la vie, prêché par le Buddha, et Foshuo yan shouming jing 佛說延壽水經 ou Sütra pour prolonger la vie, prêché par le Buddha, figurent aussi parmi les manuscrits des $\mathrm{IX}^{\mathrm{c}}$ et $\mathrm{X}^{\mathrm{c}}$ siècles ${ }^{82}$. Par ailleurs, à toute époque, la plupart des sūtra composés en Chine incorporent aux textes d'origine indienne des références aux croyances chinoises, qu'elles soient confucéennes, taoïstes ou plus généralement populaires. Les apocryphes copiés de haute époque ne sont pas plus orthodoxes que ceux copiés deux siècles plus tard. Par exemple, le Jiu jibing jing 救疾塔經 ou Sütra pour sauver de la maladie, copié en 595 (P. 4563) ${ }^{83}$, ne semble pas supérieur de ce point de vue au Fumu enzhong jing, copié en 875 (S. 4476) ${ }^{84}$. La vulgarisation d'un texte religieux dépend en effet beaucoup des besoins des fidèles, souvent les mêmes de siècle en siècle. Cela étant, la remarque de Fujieda reste vraie. Les apocryphes copiés aux $\mathrm{IX}^{\mathrm{e}}$ et $\mathrm{X}^{\mathrm{e}}$ siècles sont plus terre à terre. J'ajouterai que souvent ils ont été copiés personnellement par des fidèles soucieux de gagner des mérites, d'éviter les maladies ou désireux d'en guérir, de bénéficier d'une longue vie, de venir en aide à leurs parents défunts, etc.

Ainsi le colophon de S. 3417, placé à la suite de copies du Jiu zhuzhongsheng kunan jing 救諸衆生苦難經 ou Sütra pour délivrer tous les êtres des douleurs et catastrophes et du Xin pusa jing 新菩薩經 ou Nouveau sūtra du bodhisattva, dit qu'à nouveau victime de

78. Makita et Ochiai, 1994-2000, vol. 1.

79. Makita et Ochiai, 1994-2000, vol. $2: 657-698$.

80. Fujieda, $1973: 124$.

81. La copie de 596 est S. 635 (juan 5 du sütra), mais selon Drège (1981:320), le colophon daté est postérieur à la copie. Le manuscrit de 920 est partiellement conservé dans S. 4240 (juan 4), P. 2312 (juan 13) et S. 3691 (juan 15).

82. Ces trois textes sont énumérés dans la section d'apocryphes du catalogue de $695, T .2153(15)$, $55: 474 \mathrm{a}$.

83. Ce sūtra, écarté du canon officiel, existe en plusieurs copies à Dunhuang; celle de S. 2467 est éditée dans $T .2878,85: 1361 \mathrm{~b}-1362 \mathrm{c}$.

84. Ce sūtra est édité dans T. 2887, 85 : 1403b-1404a d'après les manuscrits S. 2034 et S. 190. Deux autres manuscrits, P. 2285 et Pékin he 河 12, et la version de l'édition coréenne ont été réédités par Makita, $1976: 50-60$. 
maladie [le fidèle] a recopié (zaixie 再寫) ces sūtra le $21^{\mathrm{c}}$ jour du septième mois de l'année cyclique dingmao 丁 形, la $5^{\mathrm{e}}$ année Qiande (967) ${ }^{85}$. S. 4479, une copie des deux mêmes textes dont le colophon esț daté de 879 , indique à la suite de la copie du premier texte, le Jiu zhu zhongsheng yiqie kunan jing 救諸衆生一切苦難經 ou Sūtra pour délivrer tous les êtres de toutes les douleurs et catastrophes, que le disciple Liu 劉 fait cette offrande d'un cœur sincère (yixin gongyang -- 心供養) et demande au Devarāja du Nord, Vaiśravana, de protéger sa demeure. Le colophon de P. 4563 indique que le Jiu jibing jing fut copié en cent exemplaires par un «disciple [du Buddha] de foi pure » (qingxin dizi 清信弟子), Tan Yonghe 談永和, qui souhaite que tous les malades guérissent et que tous les êtres bénéficient de son acte méritoire ${ }^{86}$. Le Yanluowang shouji jing 閻羅王授記經 ou Sütra concernant la prédiction du roi Yama, de S. 6230 a été copié à l'occasion de la maladie de la mère du copiste, pour obtenir la guérison rapide de celle-ci, faire qu'elle échappe à la souffrance des enfers et pour l'harmonie de toute la famille ${ }^{87}$. Le copiste du Xuming jing de P. 3115 est un maître de vinaya (lushi 律師), nommé Fayan 法晏. La copie lui a été demandée par une mère pour obtenir la guérison de sa fille malade.

Les titres des sūtra aussi sont explicites. Parmi les copies datées d'apocryphes du $\mathrm{X}^{\mathrm{e}}$ siècle, on trouve ainsi le Yanluowang shouji jing ou Sütra concernant la prédiction du roi Yama, copié en 926 (S. 6230), et le Foshuo xuming jing ou Sütra pour continuer la vie, prêché par le Buddha, copié en 901 (P. 3115) $^{88}$. Une autre copie du Xuming jing fut effectuée en 959, en même temps que celle d'un autre apocryphe, le Foshuo yan shouming jing ou Sütra pour prolonger la vie, prêché par le Buddha ${ }^{89}$, et celle d'un sütra canonique, traduit par Xuanzang 玄奖 (600-664), T.592, le Tian qingwen jing 天請問經 ou Sūtra [en réponse aux] questions d'un deva (P. 2374).

Les constatations de Fujieda sont encore plus justes pour les textes de type prophétique. Par leur contenu et leur aspect matériel, ils sont assez différents des autres catégories d'apocryphes. Ils sont plus courts, souvent copiés en plusieurs exemplaires identiques à la suite. Ces «sūtra» sont en quelque sorte des talismans, des remèdes prophylactiques pour éviter les catastrophes annoncées par ces textes. Leur titre de «jing» (sütra) excepté, ces textes n'ont rien de sütra. Prenons pour exemple le Jiu zhuzhongsheng kunan jing, le Xin pusa jing 新菩薩經 et le Quanshan jing 勸善經 ou Sūtra pour exhorter [à pratiquer] le Bien. Le contenu de ces trois textes est assez voisin. Un prophète annonce l'arrivée de mauvais présages et de désastres qui frapperont toute la famille, tout le village et toute la région. Pour éviter ces malheurs, il faut copier en plusieurs exemplaires le sūtra en question et réciter le nom d'Amituofo (Amitābha) chaque jour cent fois. Le nom du prophète est celui d'un personnage imaginaire (un vieux maître de neuf-cents ans dans le Jiu zhushongsheng kunan jing), ou même d'un personnage réel, un ministre des Tang, nommé Jia Dan 賈耽 (730-805) ${ }^{90}$, dans le Quanshan jing et le Xin pusa jing ${ }^{91}$, ou le buddha-bodhisattva (sic!) Dingguang 定光佛菩薩 (S. 2713, texte sans titre) ${ }^{92}$.

85. Pour ce qui concerne ces deux sütra, voir infra.

86. Catalogue V : 205.

87. Wang Chongmin, 1983 : 238. Le sütra est connu comme Sütra des dix rois (Shiwang jing 十王經), Teiser, 1994.

88. Le Xuming jing est mentionné pour la première fois dans le catalogue de 695 . Le texte du S. 1215 est édité dans $T .2889,85: 1405 \mathrm{a}$.

89. Ce sūtra, mentionné dans le catalogue de 695 comme apocryphe, fut écarté du canon et retrouvé à Dunhuang. Il est édité dans T. 2888, 85 : 1404a28-c29, d'après S. 2428.

90. P. 3624 lui attribue aussi la rédaction du sütra.

91. Ces deux titres sont ceux de deux versions d'un même texte.

92. Dingguang est l'une des deux traductions chinoises du nom indien Dipamkara, l'un des buddha du passé. 
Le Jiu zhuzhongsheng kunan jing et le Xin pusa jing sont souvent copiés à la suite ${ }^{93}$. Le Quanshan jing est conservé dans une version ancienne de 704, copiée sous le règne de l'impératrice Wu des Tang (S. 622) ${ }^{94}$. Il en existe au moins onze copies, dans les collections de Paris et de Londres, datées du $23^{\mathrm{e}}$ jour du premier mois de l'année jiashen 甲 申 (du cycle sexagésimal), dix-neuvième année de l'ère Zhenyuan (803). Trois portent la date du $23^{\mathrm{e}}$ jour du premier mois de l'année jiashen 甲申, mais neuvième année de l'ère Zhenyuan (793) ${ }^{95}$. Toutes ces dates sont erronées. L'année cyclique jiashen correspond plutôt à l'an 804 , pas à 803 , encore moins à 793 . D'autres copies donnent également la date de la dix-neuvième année de l'ère Zhenyuan, mais au huitième jour du premier mois ${ }^{96}$. Seul S. 1349 indique que le texte a été écrit pour la première fois (chuwen 出文) au $23^{\mathrm{e}}$ jour du premier mois de l'année cyclique jiashen, dix-neuvième année Zhenyuan (803). Il fut copié le $29^{\mathrm{e}}$ jour du mois précédent (shangyue xieliao 上月寫了), sans plus de précision ${ }^{97}$. P. 3036 indique dans le corps du texte que le décret ordonnant [de copier ce texte en grand nombre] fut publié le $23^{\mathrm{e}}$ jour du premier mois de la dix-neuvième année Zhenyuan (803) et, à la fin, que cette copie fut réalisée par Baoxuan 寶宣 en la troisième année Tianfu (938) ${ }^{98}$.

Il se peut donc que les trois dates comportant le chiffre 9 (jiu 九) au lieu de 19 (shijiu 十九) usuel soient dues à des erreurs. Mais il se peut aussi que la date indiquée à la fin du texte ne corresponde pas à la date réelle de la copie. En effet, l'année jiashen joue un rôle très important dans les prophéties taoïstes du Ve siècle. C'est l'une des années cycliques marquées par des catastrophes : déluges, massacres ${ }^{99}$. La notion se retrouve dans quelques apocryphes bouddhiques des $\mathrm{V}^{\mathrm{c}}$ et $\mathrm{VI}^{\mathrm{e}}$ siècles, notamment les textes prophétiques concernant le bodhisattva Yueguang 月光 (Candraprabha) et le futur buddha Mile 爾勒 (Maitreya) ${ }^{100}$.

\section{Copies d'apocryphes et "sūtra d'offrande "}

Le texte du manuscrit P. 2374 indique que la copie de trois sütra en 49 exemplaires pour le culte d'offrande (gongyang 供養) est due au maître de dhyāna, Huiguang 惠光 du Yongdian chanyuan 永典禪院 de Guazhou 瓜州 ${ }^{101}$. Les nombres 49 et 100 semblent en rapport avec la durée du jeûne (zhai 齋). Le colophon du manuscrit P. 2055 nous apprend que le fidèle Zhai Fengda 翟奉達, secrétaire auxiliaire du ministère des Travaux publics ${ }^{102}$, fait copier un sütra à l'occasion de chaque période de jeûne de sept jours pour en donner le mérite à son épouse décédée. Il demande aux deva et nāga, etc., soit à toutes les huit catégories de divinités, aux bodhisattva Jiuku Guanyin 救苦觀音 (Avalokiteśvara, Sauveur des douleurs) et Dizang 地藏 (Kșitigarbha), aux quatre Tianwang 天王 (Devarāja)

93. Tels P. 3117, S. 2649, 3417 (daté 967) et 4479 (daté 879). Cf. Ogasawara, 1963.

94. Il est édité sous le titre de Xin pusa jing dans T. 2917B, $85: 14662 \mathrm{~b}$.

95. Les onze manuscrits sont P. 2608, 3463, et S. 912, 1349, 3792, 3871, 4739, 4923, 4924, 5113 et P. 3624 (intitulé Quanshan wen 勸善文, mais c'est le même texte que Quanshan jing). Les trois autres sont : P. 2650, 3624 et. S. 1185(2). Voir Drège, $1981: 329$ et 318-338, où une liste de copies datées est donnée.

96. P. 3498 et un manuscrit de St. Petersburg лх 299/1313, cf. Bо Xiaoying, $1990: 81$.

97. Voir Bo Xiaoying, $1990: 82$.

98. Catalogue III : 30.

99. Kikuchi, 1996 et Mollier, $1990: 18,22,24,164$.

100. T. 2145(5), $55: 39 \mathrm{a} 15-16$ et $T .2154,55: 673 \mathrm{a} 3$ et Zürcher, 1982a : 21-22.

101. Catalogue I : 238.

102. Pour ce qui concerne la vie et l'activité religieuse de Zhai, voir Teiser, $1994: 117-121$. D'après Teiser, Zhai Fengda serait probablement né en 883 . 
et aux huit Jingang 金剛 (Vajrasattva) d'être ses témoins et d'accepter qu'il leur transfère les mérites produits par son acte ${ }^{103}$. Le colophon précise qu'une copie du Wuchang jing 無常經 ou Sūtra traitant de l'impermanence ${ }^{104}$ fut réalisée pour la cérémonie de fin du premier jeûne de sept jours (di yiqi zhai 第-..七齋); qu'une copie du Shuiyue Guanyin jing 水月觀音經 ou Sūtra de Guanyin de la lune se reflétant dans l'eau ${ }^{105}$ fut réalisée pour la cérémonie de fin du deuxième jeûne (di erqi zhai 第: 七齋); qu'une copie du Zhoumei jing 览魅經 ou Sūtra d'incantations contre les envoûtements ${ }^{106}$ fut réalisée pour la cérémonie de fin du troisième jeûne (di sanqi zhai 第三七齋); qu'une copie du Tian qingwen jing fut réalisée pour la cérémonie de fin du quatrième jeûne (di siqi zhai 第四七齋); qu'une copie du Yanluo jing 閻羅經 ou Sūtra de Yama ${ }^{107}$ fut réalisée pour la cérémonie de fin du cinquième jeûne (di wuqi zhai 第五七凨); qu'une copie du Hu zhutongzi jing 護諸童子經 ou Sütra pour protéger les enfants ${ }^{108}$ fut réalisée pour la cérémonie de fin du sixième jeûne (di liuqi zhai 第六七齋); qu'une copie du Duoxin jing 多心經 ou Sütra du cœur ${ }^{109}$ fut réalisée pour la cérémonie de fin du septième jeûne (di qi zhai 第七齋). Après sept fois sept (49) jours, la cérémonie se répéta le centième jour (bairi zhai 百日齋) où fut copié un sūtra destiné au rite de nourrir les défunts (preta), le Yulanpen jing 孟闌盆經 ou Sütra de Yulanpen (avalambana) ${ }^{110}$. Pour la cérémonie du premier anniversaire du décès (yi nian zhai 一年齋), on copia le Fomu jing 佛母經 ou Sūtra de la mère du Buddha ${ }^{11}$. Pour le troisième anniversaire (san nian zhai 三年齋), ce fut le Shan'e yinguo jing 善惡因果經 ou Sütra des causes et effets par les actes du bien et $d u \mathrm{mal}^{112}$. Le manuscrit P. 2055 contient en fait seulement ces trois derniers sūtra. La copie des sept autres sütra se trouve dans deux autres manuscrits, l'un à Tianjin, l'autre à Pékin ${ }^{113}$. Le colophon du manuscrit de Tianjin précise que la dame $\mathrm{Ma}$ 馬 décéda le premier jour du troisième mois de la cinquième année Xiande 顯德 (958) ${ }^{114}$. Les titres chinois des sütra copiés pour ces cérémonies funéraires sont significatifs ${ }^{115}$. Tout autant l'est le fait qu'au moins cinq textes sur dix sont des apocryphes.

103. Catalogue I : $39-40$ et Makita, $1976: 338-340$.

104. Le sütra semble être traduit plusieurs fois, mais seule la traduction de Yijing 義淨 (635-713) est conservée, T. 801. Cf. Teiser, $1994: 107$, n. 15.

105. Ce texte semble exister uniquement à Dunhuang, un exemplaire se trouve dans la collection du musée de la ville de Tianjin (Tianjin-shi yishu bowuguan 天津市藝術博物館), $\mathrm{n}^{\circ} 4532$. Il est édité par Fang Guangchang, 1995 : 349-350.

106. Il existe plusieurs copies avec des variantes de ce sūtra, notamment P. 3689, S. 418 et S. 2517. Les deux dernières sont éditées dans T. 2882, 85 : 1383b-1384b (cf. Catalogue IV : 170-171). Le sūtra fut également retrouvé au Nanatsu-dera : Makita et Ochiai, 1994-2000, vol. II : 701-742.

107. Ceci est un titre abrégé du Yanluowang shouji jing, mentionné ci-dessus, p. 691.

108. T. 1028A, $19: 741$ b19-742c6 traduit par Bodhiruci au VIc siècle.

109. Titre abrégé du Poruo poluomiduo xin jing 般若波羅密多心經 (Prajñāpāramitā-hrdayasütra), (T. 251-255).

110. La traduction de ce sūtra (T. 685) est attribuée à Fahu 法護 (Dharmarakṣa, 265-313), mais l'attribution n'est pas sûre, $c f$. Teiser, $1988: 48-55$.

111. Ce sütra fabriqué en Chine se trouve seulement à Dunhuang, en deux versions légèrement différentes, P. 4576, S. 1371, 2084, 3306, 6960, etc. S. 2084 est édité dans T. 2919, 85: 1463a18-b23 (non S. 6960 et T. 2910 comme indiqué par Durt, $1995: 8$, n. 22). Cf. Catalogue V: 211. Le texte est commenté par Teiser, $1994: 113-114$ et Durt, $1995: 8-9$.

112. Le texte édité dans le Nihon Zokuzōkyō ou Suite du Canon compilé au Japon complété par un manuscrit de Dunhuang dans l'ancienne collection de Nakamura Fusetsu, a servi de base pour l'édition de $T$. $2881,85: 1380 \mathrm{~b}-1383 \mathrm{~b}$.

113. Teiser, $1994: 102$.

114. Teiser, $1994: 102-103$ et Fang Guangchang, $1995: 351-352$.

115. Teiser, $1994: 102-121$ et 156 sqq. 
Ces sūtra copiés à l'occasion d'une cérémonie sont dits les "sūtra d'offrande " ou «sūtra de culte» (gongyang jing 供養經) ${ }^{116}$. Appartiennent aussi à la catégorie des «sütra d'offrande » les sütra dont le colophon n'indique pas qu'ils ont été copiés à l'occasion d'une cérémonie, mais qui répondent à un besoin précis (prolonger la vie, guérir de maladie, avoir une bonne renaissance, etc.) donnant lieu généralement à une cérémonie. Nous avons parlé de ces sütra plus haut (p. 690-691). Ces sütra de culte sont souvent des «sütra» de la fabrication chinoise, copiés par le croyant pour ses besoins propres. Cet aspect personnel, exprimant un besoin religieux très différent des entreprises officielles de copies, semble avoir été sous-estimé par Fujieda.

\section{Spécificités de Dunhuang}

Il existe à Dunhuang des manuscrits comportant plusieurs textes à la suite sans colophon ou indication de vœux précis ${ }^{117}$. Ils se présentent en général sous forme de petits livrets ${ }^{118}$. La plupart peuvent être datés du $X^{\mathrm{e}}$ siècle par le support et l'écriture. P. 3932 en est un bon exemple. Il a en frontispice un dessin de Guanyin à la branche de saule audessus d'un couple de donateurs ${ }^{119}$. Il contient une copie de cinq sütra suivis de deux courts mantra dits respectivement de Maitreya (Cishi zhenyan 慈氏畺言) et de "purification des [mauvaises] actions causées par les paroles" (jing kouye zhenyuan 浄口業畺言). Les cinq sūtra en question sont: 1. le chapitre 25 du Sütra du Lotus (Sūtra de Guanyin) ; 2. le Poruo xinjing 般若心經, Prajñāpāramitā-hrdaya-sūtra ; 3. le Foshuo xuming jing 佛說續命經 ou Sütra pour continuer la vie, prêché par le Buddha; 4. le Foshuo Dizang pusa jing 佛說地藏菩薩經 ou Sūtra du bodhisattva Dizang (Kșitigarbha), prêché par le Buddha; 5. le Foshuo jiebaisheng yuanjia tuoluoni jing 佛說解百生怨家陀羅尼經 ou Sūtra prêché par le Buddha [contenant] la dhārañi pour dissoudre la haine accumulée depuis cent naissances ${ }^{120}$. Trois de ces cinq textes sont des apocryphes. Le quatrième texte, Sütra du bodhisattva Dizang, n'est pas l'un des sütra au nom du bodhisattva Dizang connus par ailleurs, qu'ils soient canoniques ou apocryphes. Il ne se trouve qu'à Dunhuang ${ }^{121}$. Le Sütra prêché par le Buddha [contenant] la dhäran̄i pour dissoudre la haine accumulée depuis cent naissances, inédit, de 150 mots environ, n'existe également qu'à Dunhuang, mais il est représenté par plusieurs manuscrits (P. 3824(3), 2169 ro, S. 2900, 4223, 4271, 4431, 5235, etc.). Ces séries de sütra enchaînés regroupés en petits livrets se trouvent encore notamment dans les manuscrits S. 5447, $5450,5458,5531,5534,5581,5585,5646$, etc ${ }^{122}$.. Beaucoup sont de fabrication chinoise, par exemple, ceux qui peuvent prolonger la vie (Xuming jing et Yan shouming jing); ceux qui protègent le corps et guérissent de la maladie (Hu shenming jing et Jiu jibing jing); ceux qui sont en rapport avec la croyance aux enfers (Yanluowang jing et Dizang pusa jing) ; celui de la dhārañi pour dissoudre la haine (Jie baisheng yuanjia tuoluoni jing); celui qui décrit les bienfaits des parents (Fumu enzhong jing); celui qui raconte l'histoire

116. L'expression est souvent utilisée par nos collègues japonais. Elle se trouve même parfois dans certains colophons, par exemple sur une copie du Hu shenming jing 護身命經 ou Sütra pour protéger la vie, de l'ancienne collection de Nakamura Fusetsu aujourd'hui conservée au musée de calligraphie de Tokyo et éditée dans $T$. 2866, $85: 1326 \mathrm{~b}-1327 \mathrm{a}$.

117. Ces copies sont appelées par Makita rensha-kyō 連寫經, « sūtra-copies enchaînées » (1976: 39).

118. Pour ce qui concerne les manuscrits sous forme de livrets, voir Drège, 1979.

119. Ce frontispice est reproduit dans Giès et Cohen, $1995: 361$ et Drège, $1999: 111$.

120. Catalogue IV : 423-424.

121. Wang-Toutain, 1998 : 145-150. Selon Wang-Toutain (p. 146), la copie du Dizang pusa jing éditée dans $T$. 2909, $85: 1455$ b24-c12 est S. 6257, et non S. 197 comme indiqué.

122. Teiser, $1994:$ : 273-274. 
de la mère du Buddha (Fomu jing). Ce sont très certainement des ouvrages recopiés pour être utilisés dans le culte.

Makita souligne qu'une vingtaine de «sūtra» chinois ne sont connus qu'à Dunhuang. Il a raison de penser que ces sūtra chinois y ont été fabriqués ${ }^{123}$. Le chiffre donné par Makita est sans doute à vérifier. Mais une chose est sûre : à Dunhuang, il existe, outre ces livrets de sūtra enchaînés, beaucoup de textes rituels et de manuels qui ont été rédigés localement ou transmis seulement dans cette région. Ils sont inconnus ailleurs en Chine. Mais ils correspondent à des pratiques ésotériques et ont été utilisés par des groupes certainement plus restreints que les sütra populaires que je viens d'évoquer. Ces textes rituels étaient à l'usage des religieux, pas des simples laïcs.

\section{Textes de Dunhuang datant de la domination tibétaine}

Les textes rituels ou tantra particuliers à Dunhuang sont souvent dits par nos collègues japonais «s $\bar{u} t r a$ traduits en chinois mais non transmis en Chine centrale » (chügen miden kanyaku kyōden 中原未傳漢譯經典) ${ }^{124}$. Dunhuang fut sous contrôle tibétain de 781 à 848. Des traducteurs de cette époque, tel le moine tibétain Facheng 法成 appelé Chos-grub en tibétain, étaient parfaitement bilingues ${ }^{125}$. P. Demiéville et R. A. Stein ont montré que le bouddhisme chinois, notamment celui de l'école Chan, a eu une grande influence sur le bouddhisme tibétain ${ }^{126}$. Mais les rapports entre les bouddhismes chinois et tibétain semblent avoir été plus complexes. À Dunhuang, coexistaient en effet des traditions canoniques et un système de pratiques rituelles propres à cette région. Dans beaucoup de peintures murales et sur les bannières rituelles mobiles, on constate le mélange de deux traditions classées bien plus tard comme, respectivement, sino-japonaise et indo-tibétaine.

À côté des sütra traduits par Facheng ${ }^{127}$, il existe parmi les manuscrits de Dunhuang quelques sūtra qui auraient été traduits à Anxi (Koutcha) durant le VIII siècle. Ainsi le Huixiang lun jing 迴向輪經 ou Sütra de la roue de transferts-des-mérites (parinämana), aurait été traduit par un moine khotanais nommé Shiluodamo P羅達摩 (Śiladharma ?) dans le monastère Longxing 龍興 寺 de Beiting 北庭 (Baishaliq) (P. 3918) ${ }^{128}$. Shiluodamo traduisit également le Shili jing 十力經 ou Sütra des dix forces (bala) (T. 780, $17: 715 \mathrm{c}-$ $717 \mathrm{c}$ ). Le moine Wukong 悟空 (VIII ${ }^{\mathrm{e}}$ siècle) se procura ces deux traductions chinoises, plus une nouvelle traduction du Shidi jing 十地經 ou Sütra des dix Terres (bhümi) ${ }^{129}$. Ces trois traductions, 11 rouleaux et 129 feuilles en tout, étaient rangées dans une même enveloppe. Comme elles ne figuraient pas dans les catalogues, Wukong eut peur qu'elles soient traitées comme les sūtra faux ou douteux (yiwei jing 疑偏經). Il demanda qu'elles soient incorporées au catalogue de l'ère Zhenyuan (794), suite de celui de Zhisheng (730) ${ }^{130}$. Les trois traductions figurent effectivement dans la liste du canon officiel de 794 (T. 2156, 55: 769a7-12). Mais le Huixiang lun jing est absent du canon dit « des trois éditions » (des Song, des Yuan et des Ming). Le texte édité dans le Taishō est celui de l'édition coréenne (T. 998, $19: 577 \mathrm{a}-578 \mathrm{a})$. Le texte de Dunhuang est donc plus ancien.

123. Makita, $1976: 38-39$.

124. Ueyama, 1972 et Shirasu, 1974.

125. Demiéville, 1952 : 20-21, n. 5 ; Ueyama, 1967-68.

126. Démiéville, 1952 et 1979 ; R. A. Stein, 1971 et 1984.

127. Pour les œuvres de Facheng, voir Ueyama, 1967-68.

128. Le sütra se trouve sur les manuscrits P. 2008, 3918, S. 2540, 1385 et $l i$ 麗 74 de Pékin, etc., cf. Shirasu, 1974 : 39-43. À propos de l'appellation «Longxing si » pour les monastères bouddhiques, voir Kuwayama, $1992: 231-235$.

129. Pour le voyage de Wukong, voir Lévi et Chavannes, 1895.

130. T. 2089, 51 : 980c-981b ; voir aussi le texte concernant le Shili jing, $T$. 780, $17: 715 \mathrm{c}-717 \mathrm{~b}$. 
Un autre sūtra, Foshuo jingangtan guangda qingjing tuoluoni jing 佛說金剛壇 廣大清淨陀羅尼經 ou Sūtra prêché par le Buddha [contenant] la dhāranì vaste et pure de l'autel de diamant, dont nous possédons un seul exemplaire copié à la suite du Huixiang lun jing sur le manuscrit P. 3918, aurait également été traduit à Anxi (Koutcha) par un śramana Tanqing 曼倩 en 753 [littéralement quarante ans avant la neuvième année Zhenyuan (793)]. Sa diffusion serait due à un haut fonctionnaire devenu moine, nommé Lizhen 利真. La postface raconte les miracles produits pour ceux qui croient à ce sütra et les punitions pour ceux qui n'y croient pas ${ }^{131}$. Tanqing est inconnu par ailleurs. Le contenu du sütra est similaire à celui d'un autre texte de dhäranī dont deux versions figurent dans le canon du Taishō: T.1344, Jingang shangwei tuoluoni jing 金剛上味陀羅尼經 ou Sütra de la dhārañi de la saveur suprême de diamant (traduit par Buddhaśānta en 525), et T. 1345, Jingangchang tuoluoni jing 金剛場陀羅尼經 ou Sütra de la dhārañi de l'aire de diamant (traduit en 587 par Jñānagupta). Il est probable que P. 3918 est une troisième traduction de ce texte ou une composition faite à partir des deux versions.

Il existe d'autres sītra de dhāranì inédits à Dunhuang. Ils datent pour la plupart du $\mathrm{X}^{\mathrm{e}}$ siècle. Le Dafoding rulai dingji baigai tuoluoni shenzhou jing 大佛頂如來頂彭白蓋陀 羅尼神咒經 ou Sūtra de la formule divine, dhärani du parasol blanc de la protubérancecrânienne du Buddha, est copié avec d'autres sütra dans le manuscrit P. $3916^{132}$. La première partie de ce texte est une translittération de dhâranī, proche de la translittération d'une autre dhāranì dite également du parasol de la protubérance crânienne du Buddha, Dafoding rulai fangguang xidanduobotan tuoluoni 大佛頂如來放光悉柦多缽坦陀羅尼 (Sarvatathāgata-usṇissa-sitātapatrā-dhāran̄i ), attribuée au grand maître ésotérique du VIII siècle, Amoghavajra (T. 944A). La seconde partie du texte de Dunhuang ressemble au texte d'un autre sūtra de dhäranī dit aussi du parasol de la protubérance crânienne du Buddha, Foding dabaisangai tuoluoni jing 佛頂大门傘蓋陀羅尼經 (Sitātapatra-dhāranī), traduit par un moine tangut, Shalopa (1259-1314), T. 976. Nous pouvons également supposer que le sūtra de P. 3916 est une ancienne traduction de la Buddha-usnissasitātapatrā-dhāranī.

Le Jingang jun jing 金剛峻經 ou Sütra de l'escarpement de diamant fut certainement fabriqué à Dunhuang. Il existe en trois versions qui ne sont pas tout à fait identiques ${ }^{133}$. Titre et contenu s'inspirent du Jingang ding jing 金剛頂經 ou Sūtra de la pointe de diamant, ou encore Vajraśekhara-sütra, l'un des plus importants tantra de la tradition sino-japonaise ${ }^{134}$. Selon P. 3913, le Jingang jun jing de Dunhuang aurait été traduit par Amoghavajra (705-774), mais la date de 899 figure dans le corps du texte prouve que la rédaction en est postérieure d'au moins 120 ans à la mort de son traducteur prétendu. Ce tantra indique plus de 40 façons d'établir et utiliser le mandala de l'autel d'ordination (jietan 笺壇). La nouveauté de ce tantra rédigé directement en chinois est dans les noms donnés aux divinités installées sur le mandala. Les noms indiqués pour les bodhisattva et vajrasattva sont totalement inconnus des sources canoniques, mais ils sont explicites. $\mathrm{Ce}$ sont des noms de pratiques rituelles nécessaires à l'établissement de mandala: “Confession» (chanhui 懺悔), «Purifie-la-Terre» (jingdi 海地), « Délimite-l'aire » (jiejie 結界), “Purifie-l'aire » (jingjie 淨界) ${ }^{135}$. Le fait que le mandala soit occupé et protégé par des divinités dont les noms évoquent les actes principaux du rite avant même que celui-ci

131. Ueyama, 1972 et Catalogue IV : 407-408.

132. Catalogue IV : 405.

133. Ce sont P. 3913 , S. $2144 v^{\circ}$ et un manuscrit de la collection de Pékin, dong 冬 74.

134. Sakano, 1976 ; Hatta, 1985 : 83-194 ; et Horiuchi, 1996.

135. Cf. Hirai, 1977 ; Kuo, 1998 : 240-244 ; Tanaka Kimiaki, 2000. 
n'ait commencé semble répondre à une volonté d'efficacité. Le tantra pourrait relever de l'école Chan, car sa dernière section est une chronique des patriarches de cette école en Inde et en Chine ${ }^{136}$.

Ce tantra fut utilisé, car des divinités portant les mêmes noms se retrouvent sur un rouleau de dessins de mandala de Dunhuang (P. 2012) ${ }^{137}$. Les couleurs des divinités siégeant aux points cardinaux sont écrites sur les dessins, mais ne correspondent pas à celles que l'on connaît dans la tradition indo-tibétaine ou sino-japonaise. Peut-on dire que ce traité rituel et ces dessins de mandala sont particuliers à Dunhuang et reflètent des aspects du tantrisme chinois local à l'époque où bouddhistes chinois et tibétains habitaient côte à côte ? D'autres recherches permettront de confirmer ou d'infirmer ce point de vue.

\section{Conclusion}

La science occidentale applique donc le terme d'apocryphe à des textes très divers. L'appellation, qui traduit en fait l'expression «s sutra faux et douteux » des catalogueurs chinois, s'applique à des traductions d'origine inconnue, à des traductions mal faites, à des florilèges malhabiles autant qu'à des textes composés directement en chinois à Dunhuang ou ailleurs en Chine. On trouve dans tous, dans des proportions diverses mais plus importantes en général que dans les sütra dits authentiques, l'influence de croyances religieuses chinoises : morale confucéenne, préoccupations taoïstes, craintes et espérances populaires.

Vue de plus près, la catégorie "apocryphe » mêle des textes très différents, certains largement et longuement diffusés, d'autres qui ne connurent aucun succès. La nature de leur contenu change avec le temps. 11 y a loin des sutra, orthodoxes quoique apocryphes, $\mathrm{du} \mathrm{V}^{\mathrm{e}}$ siècle aux textes populaires et tantra du $\mathrm{X}^{\mathrm{e}}$ siècle. On note avec intérêt que beaucoup d'apocryphes sont utilisés dans des rituels à visée très modeste, pour obtenir une guérison, échapper à un désastre, faire acte de piété filiale. Ils répondent aux besoins de la masse des fidèles chinois : tous ne voulaient pas devenir des bodhisattva. Utilisés dans des rituels financés par les laïcs à leur propre usage, ils devaient coller au plus près possible du quotidien de la piété chinoise. Ce à quoi les textes fabriqués sur place spécialement à cet usage étaient évidemment le mieux adaptés.

Fort différents sont les tantra de Dunhuang. Ils correspondent aussi à une pratique locale, mais ésotérique et réservée à des initiés. Ils permettent de soupçonner l'existence d'une pratique d'origine locale, qui n'a pas été importée d'ailleurs en Chine et n'y a pas non plus essaimé. Ce n'est pas le cas des autres apocryphes de Dunhuang. Ceux dont on n'a pas d'autres exemples en Chine n'étaient pas nécessairement d'origine locale et leur diffusion n'était pas nécessairement limitée à Dunhuang. Le hasard ou des circonstances particulières sont peut-être responsables du fait que seule la grotte aux manuscrits en ait conservé des copies.

La production d'apocryphes continue après le $X^{\mathfrak{c}}$ siècle. Il existe bien d'autres textes bouddhiques que ceux contenus dans le canon des Song, des Ming, des Qing. Stricto sensu, ce sont des apocryphes, des sütra faux et douteux, même si on ne les appelle plus ou pas encore ainsi.

136. Cf. Tanaka Ryōsho, 1974-77; Ueyama, 1981.

137. Jao, 1978, fasc. I : 28 et III : pl. LVI-LXV ; Kuo, 1998. 


\section{Bibliographie *}

Bo Xiaoying 薄小瑩

1990 Dunhuang yishu hanwen jinian juan biannian 敦煌遺書漢文紀年卷編年 [Chronologie des manuscrits chinois datés de Dunhuang], Changchun 長春, Changchun chubanshe 長春出版社.

BOKENKAMP, Stephen R.

1983 "Sources of the Ling-pao Scriptures", in Tantric and Taoist Studies in Honour of R. A. Stein, Michel Strickmann éd., Bruxelles, Institut belge des hautes études chinoises (Mélanges chinois et bouddhiques, vol. XXI), vol. 2 : 434-486.

1990 "Stages of Transcendence: The Bhūmi Concept in Taoist Scripture », in Chinese Buddhist Apocrypha, Robert E. Buswell éd., Honolulu, University of Hawai'i Press, p. 119-147.

BROUGH, John

1950 "Thus Have I Heard ... », Bulletin of the School of Oriental and African Studies, 8/2, p. 416-426.

BURNOUF, Eugène

1844 Introduction à l'histoire du bouddhisme indien, Paris, Imprimerie royale, [rééd. Paris, Maisonneuve, 1876].

1852 Le lotus de la Bonne Loi, Paris, Imprimerie nationale, [rééd. avec une préface de S. Lévi, en 2 vol., Paris, Maisonneuve, 1925].

BUSWELL, Robert E.

1990 "Introduction: Prolegomenon to the Study of Buddhist Apocryphal Scriptures ", in Chinese Buddhist Apocrypha, Robert E. Buswell éd., Honolulu, University of Hawai'i Press, p. 1-30.

CALlOWAY, Brian

"Thus Have I Heard: At One Time ... », Indo-Iranian Journal, 34/2, p. 87104.

CAtalogue I

1970 Catalogue des manuscrits chinois de Touen-houang. Fonds Pelliot chinois, vol. I ( ${ }^{\text {os }}$ 2001-2500), par Jacques Gernet et Wu Chi-yu, Paris, Bibliothèque nationale.

\section{Catalogue III}

1983 Catalogue des manuscrits chinois de Touen-houang. Fonds Pelliot chinois de la Bibliothèque nationale, vol. III ( $\left.\mathrm{n}^{\mathrm{os}} 3001-3500\right)$, sous la direction de Michel Soymié, Paris, Éditions de la Fondation Singer-Polignac.

\section{Catalogue iV}

1991 Catalogue des manuscrits chinois de Touen-houang. Fonds Pelliot chinois de la Bibliothèque nationale, vol. IV ( $\left.\mathrm{n}^{\text {os }} 3501-4000\right)$, sous la direction de Michel Soymié, Paris, EFEO.

\footnotetext{
* Lorsque les titres chinois et japonais sont traduits par l'auteur de l'ouvrage qui les utilise ou les cite, cette traduction est ici donnée entre parenthèses (). Lorsque qu'elle est de moi, elle est donnée entre crochets [ ].
} 


\section{Catalogue V}

1995 Catalogue des manuscrits chinois de Touen-houang. Fonds Pelliot chinois de la Bibliothèque nationale, vol. V ( $\left.\mathrm{n}^{\text {os }} 4001-6040\right)$, sous la direction de Michel Soymié, 2 t., Paris, EFEO.

CHEN Guofu 陳國符

1963 Daozang yuanliu kao 道藏源流㪀 [L'origine du canon taö̈que], Beijing, Zhonghua shuju.

DAVIDSON, Ronald M.

1990 «An Introduction to the Standards of Scriptural Authenticity in Indian Buddhism ", in Chinese Buddhist Apocrypha, Robert E. Buswell éd., University of Hawaii Press, p. 291-325.

DE GROOT, J. J. M.

1893 Le code du Mahāyäna en Chine, son influence sur la vie monacale et sur le monde laïque, Amsterdam, Johannes Müller [réimpr. 1967].

DE VISSER, M. W.

1935 Ancient Buddhism in Japan. Sütra and Ceremonies in Use in the Seventh and Eighth Centuries A.D. and Their History in Later Times, Leiden, E. J. Brill.

DEMIÉVILLE, Paul

1929 "Sur l'authenticité du Ta Tch'eng k'i sin louen », Bulletin de la Maison Franco-Japonaise, II/2, p. 1-78.

1930 «Bosatsukai » (Bodhisattvaśīla), Hōbōgirin, Dictionnaire encyclopédique du bouddhisme d'après les sources chinoises et japonaises, fasc. 2, p. 142a146b.

1952 Le concile de Lhasa. Une controverse sur le quietisme entre bouddhistes de l'Inde et de la Chine au VIII' siècle de l'ère chrétienne, Paris, Presses universitaires de France (Bibliothèque de l'Institut des hautes études chinoises, vol. VII).

1954-56 «Langue et littérature chinoises », Annuaire du Collège de France 19541955, p. 246-249 et 1955-1956, p. 237-241 [repris dans Choix d'études sinologiques, Leiden, E. J. Brill, 1973, p. 148-157].

1979 «L'introduction au Tibet du bouddhisme sinisé d'après les manuscrits de Touen-houang: analyse de récents travaux japonais ", in Contributions aux études sur Touen-houang, sous la direction de Michel Soymié, Genève/Paris, Droz, p. 1-16.

DRĖGE, Jean-Pierre

1979 "Les cahiers des manuscrits de Touen-houang», in Contributions aux études sur Touen-houang, sous la direction de Michel Soymié, Genève/Paris, Droz, p. 17-28.

1981 «Papiers de Dunhuang, essai d'analyse morphologique des manuscrits chinois datés ", T'oung Pao, 62/3-5, p. 305-360.

1991 Les bibliothèques en Chine au temps des manuscrits (jusqu'au Xe siècle), Paris, EFEO (PEFEO, 161).

1999 «Du texte à l'image : les manuscrits illustrés », in Images de Dunhuang. Dessins et peintures sur papier des fonds Pelliot et Stein, Jean-Pierre Drège éd., Paris, EFEO (Mémoires archéologiques, 24), p. 105-167.

DURT, Hubert

1996 «L'apparition du Buddha à sa mère après son nirvāṇa dans le Sūtra de mahāyāna et le Sūtra de la mère du Buddha », in De Dunhuang au Japon. 
Études chinoises et bouddhiques offertes à Michel Soymié, textes réunis par Jean-Pierre Drège, Genève, Droz, p. 1-24.

FANG Guangchang 方廣錩

1991 Fojiao Dazangjing shi (ba-shi shiji) 佛教大藏經史 (八-十世紀) [Histoire du canon bouddhique (chinois) du VIII au $X^{e}$ siècle], Beijing, Zhongguo shehui kexue chubanshe 中國社會科學出版社.

1995 Zangwai fojiao wenxian 藏外佛教文獻 [Documents bouddhistes non canoniques], Fang Guangchang éd., Beijing, Zongjiao wenhua chubanshe 宗教文化出版社, vol. I.

FUJIEDA Akira 藤枝晃

1973 "The Tun-huang Manuscripts", in Essays on the Sources for Chinese History (Fitzgerald Festschift), Donald D. Leslie, Colin Mackerras et Wang Gung-wu éd., Canberra, Australian National University, p. 120-128.

GIĖs, Jacques \& COHEN, Monique

1995 Sérinde, terre de Bouddha. Dix siècles d'art sur la Route de la Soie, (Catalogue de l'exposition aux Galeries nationales du Grand Palais, Paris 24 octobre 1995-19 février 1996), Paris, Éditions de la Réunion des musées nationaux.

GRONER, Paul

1990 «The Fan-wang ching and Monastic Discipline in Japanese Tendai: A Study of Annen's Futsu jubosatsukai kōshaku », in Chinese Buddhist Apocrypha, Robert E. Buswell éd., Honolulu, University of Hawai'i Press, p. 251-290.

HATTA Yukio, 八田幸雄

$1985 J \bar{u} y \bar{o}$ mikkyō kyōgi kaisetsu 主要密教經軌解說 [Explication des principaux sūtra et tantra du bouddhisme ésotérique], Tōkyō, Hirakawa shuppansha.

HAYASHIYA Tomojirō 林屋友次郎

1941 Kyōroku kenky $\bar{u}$ 經錄研究 [Étude sur les catalogues bouddhiques], Tōkyō, Iwanami shoten.

HINÜBER, Oskar von

1968 Studien zur Kasussyntax des Pāli, besonders des Vinaya-Pițaka, München, Kitzinger.

HIRAI Yūkei 平井宥慶

1977 «Tonkō shutsudo gigikyō bunken yorimita mikkyō to zen » 敦煌出土 疑倸經文獻 より見た密教と樿 [Tantrisme et Chan dans les sūtra apocryphes de Dunhuang], in Katō Shōichi sensei koki kinen ronbunshū: Bukkyō to Girei 加藤章一先生古稀記念論文集：佛教と儀禮 [Mélanges pour commémorer le soixante-dixième anniversaire du Professor Katō Shōichi : bouddhisme et rites], Tōkyō, Kokusho kankōkai, p. 139-162.

1979 «Tonkō bunken shiryō yori mita mikkyō no iddammen » 敦煌文獻資料より見た密教の一斷面 [Un aspect du tantrisme vu à travers des textes et documents de Dunhuang], Toyoyama kyōgaku taikai jiyō 豐山教學大會紀要 [Mémoire des enseignements de Toyoyama], 7, p. 101112.

1981 «Tonkō bunken to Chūgoku bukkyō girei no mondai »敦煌文獻と中 國佛教儀禮の問題 (Literature in Tun-huang and the Buddhist Cult in China), Mikkyōgaku kenkyū 密教學研究 (The Journal of Esoteric Buddhist Study), 13, p. 124-139. 
1984 《Tonkō bon mikkyō gikyōten kō 》敦煌本密教疑經典考 (On NonCanonical Scriptures of Esoteric Buddhism as Found in Tun-huang MSS), Mikkyō bunga 密教文化 (Quarterly Reports on Esoteric Buddhism), 150, p. 53-73.

HORIUCHI Kanjin 堀內寬仁

1996 Kongōchōgyō no kenkyū 全剛頂經の研究 [Études sur le Vajraśekharasūtra], Kyōto, Hōzōkan [Recueil d'articles, vol. I].

INOKUCHI Taijun 井の口泰涼

1959 «Sutain-bon ni yoru Tonkō shutsudo gibō Butsumyōkyō no ikkōsatsu » スタイン本による 敦煌出十俘妄佛名經の -..考察 [Étude sur le sūtra apocryphe, Sūtra des noms de buddha de Dunhuang dans la collection Stein], Indogaku bukkyōgaku kenkyū 印度學佛教學研究 (Journal of Indian and Buddhist Studies), 7/2, p. 211-214.

1963 《Tonkō bon Butsyumyōkyō no shokeitō »敦煌本佛名經の諸系統 (The Filiation of the Tunhuang Text of the Buddhanāma sūtra (Fo-ming-ching)), Tohōgakuhō 東方學報 (Journal of Oriental Studies), 35, Kyōto, p. 397-437.

JAO Tsong-Yi

1978 Peintures monochromes de Dunhuang (Dunhuang Baihua), (manuscrits reproduits en fac-similé, d'après les originaux inédits conservés à la Bibliothèque nationale de Paris, avec une introduction en chinois par Jao Tsong-yi, adaptée en français par Pierre Ryckmans. Préface et appendice par Paul Demiéville), 3 fasc, Paris, EFEO (Mémoires archéologiques, 13).

KIKUCHI Noritaka 菊地章太

1996 «Kanshin daisui kō - Tōshin makki no zūshin teki Dōgyō to sono keifu » 甲申大水考一東㚙末期圖識的道經とその系譜 (The Eastern Jin Prophecies on Floods of the Year Jiashen Described in Daoist Scriptures), Tōhō shūkyō 東方宗教 (The Journal of Eastern Religions), 87, p. 1-20.

KuO Li-ying (Kuo Liying)

1994a Confession et contrition dans le bouddhisme chinois du ve au X $X^{e}$ siècle, Paris, EFEO (PEFEO, 170).

1994b «Divination, jeux de hasard et purification dans le bouddhisme chinois. Autour d'un sūtra apocryphe chinois, le Zhanchajing ", in Bouddhisme et cultures locales : quelques cas de réciproques adaptations, Fukui Fumimasa et Gérard Fussman éd., Paris, EFEO (Etudes thématiques, 2), p. 145-167

1995a "La récitation des noms de buddha en Chine et au Japon », T'oung Pao, 81, p. $230-268$.

$1995 \mathrm{~b}$ «[Comptes rendus de] Ochiai Toshinori, Manuscripts of Nanatsu-dera. A Recently Discovered Treasure-House in Downtown Nagoya, Kyoto, 1991 et Makita Tairyō et Ochiai Toshinori (éd.), Nanatsu-dera koitsu kyōten kenkyū sōsho (The Long Hidden Scriptures of Nanatsu-dera, Research Series) I : Chügoku senjutsu kyōten (Scriptures Composed in China, volume I), Tōkyō, Daitō shuppansha, 1994 », BEFEO, 82, p. 414-420.

1998 «Mandala et rituel de confession à Dunhuang », BEFEO, 85, p. 227-256.

1999 «À propos de Makita Tairyō et Ochiai Toshinori, éd., Nanatsu-dera koitsu kyōten kenkyū sōsho (The Long Hidden Scriptures of Nanatsu-dera, Research Series) II : Chūgoku senjutsu kyōten (sono ni) (Scriptures Composed in China, vol. II), Tōkyō, Daitō shuppansha, 1996 », BEFEO, 86, p. 522-531. 
KUWAYAMA Shoshin (éd.) 桑山正進

$1992 E c h \bar{o}$ Ögotenjikkokuden kenkyū 慧超往五天竺國傳研究 (Huichao's Wang Wu-Tianzhuguo zhuan. Record of Travels in Five Indic Regions, Translation and Commentary), Shoshin Kuwayama éd. avec le concours de Minoru Inaba, Kyōto, Kyoto University (Institute for Research in Humanities).

LAI, Whalen

1987 «The Earliest Folk Buddhist Religon in China : Ti-wei Po-li ching and Its Historical Significance ", in Buddhist and Taoist Practice in Medieval Chinese Society, Honolulu, University of Hawai'i Press, p. 11-35.

1990 "The Chan-ch'a ching: Religion and Magic in Medieval China », in Chinese Buddhist Apocrypha, Robert E. Buswell éd., Honolulu, University of Hawai'i Press, p. 175-206.

LAMOTTE, Étienne

1944-80 Le traité de la grande vertu de sagesse de Nāgārjuna (Mahāprajñāpāramitā-śāstra), t. I [1944, réimpr. 1966 et 1981], t. II [1949, réimpr. 1967 et 1981], t. III [1970], t. IV [1976], t. V [1980], Louvain-La-Neuve, Université de Louvain (Institut orientaliste).

1947 "La critique d'authenticité dans le bouddhisme", in India Antiqua [Mélanges J. Ph. Vogel], Leiden, E. J. Brill, p. 213-222

1949 «La critique d'interprétation dans le bouddhisme », Annuaire de l'Institut de Philologie et d'Histoire Orientales et Slaves de l'Université Libre de Bruxelles, 9 [Mélanges H. Grégoire], p. 341-361.

LÉVI, Sylvain \& CHAVANNES, Édouard

1895 «L'Itinéraire d'Ou-k'ong (751-790) », Journal asiatique, 6, p. 341-384.

LEWIS, Mark Edward

1990 «The Suppression of the Three Stages Sect: Apocrypha as a Political Issue ", in Chinese Buddhist Apocrypha, Robert E. Buswell éd., Honolulu, University of Hawai'i Press, p. 207-238.

MAKITA Tairyō 牧田諦亮

1971 "The Ching-tu san-mei ching and the Tunhuang Manuscripts », East and West , 21/3-4, p. 351-361.

1976 Gikyō kenkyu 疑經研究 [Recherches sur les sūtra apocryphes], Kyotō, Université de Kyōto (Jinbun kagaku kenkyūjo).

MAKITA Tairyō牧田諦亮 \& OCHIAI Toshinori 落合俊典 (éd.)

1994-2000 Nanatsu-dera kōitsu kyōten kenkyū sōsho 七寺古逸經典研究叢書 (The Long Hidden Scriptures of Nanatsu-dera, Research Series), Tokyo, Daitō shuppansha, vol. I (1994), vol. II (1996), vol. III (1995), vol. IV (1999), vol.V (2000) et vol. VI (1998).

\section{MoCHIZUKI Shinkō 望月信亨}

1946 Bukkyō kyōten seritsu shiron 佛教經典成立史論 [Étude de la formation des sūtra bouddhiques], Kyōto, Hōzōkan.

MOLLIER, Christine

1990 Une apocalypse taoüste du ve siècle. Le livre des incantations divines des grottes abyssales, Paris, Collège de France (Institut des hautes études chinoises). 
MüLLER, F. Max (éd.)

1881 Buddhist Texts from Japan, Anecdota Oxoniensia, Texts, Documents, and Extracts chiefly from Manuscripts in the Bodleian and other Oxford Libraries, Aryan Series, vol. I, part I, Oxford, The Clarendon Press.

Müller, F. Max \& Bunyiu NANJio (éd.)

1883 Sukhāvatī-vȳuha, Description of Sukhāvatī, The Land of Bliss, Anecdota Oxoniensia, Texts, Documents, and Extracts chiefly from Manuscripts in the Bodleian and other Oxford Libraries, Aryan Series, vol. I, part II, Oxford, The Clarendon Press.

OCHIAI Toshinori

1991 The Manuscripts of Nanatsu-dera, with related remarks by Makita Tairyō et Antonino Forte, Silvio Vita trad. et éd., Kyoto, Italian School of East Asian Studies.

1996 «Découverte de manuscrits bouddhiques chinois au Japon », BEFEO, 83, p. 368-375.

OGASAWARA Senshū 小篮原宣秀

1963 《Tonkō bon Kanzen kyō wo megurite» 敦煌本勸善經をめぐリて[À propos du Quanshan jing de Dunhuang], Tōhō shūkyō 東方宗教 (The Journal of Eastern Religions), 22, p. 1-13.

ONO Gemmyō 小野立邚”

1936 Butten sōron 佛典總郭 [Discussion d'ensemble concernant les textes bouddhiques], Bussho kaisetsu daijiten 佛書解說大辭典 [Grand dictionnaire des auvres bouddhiques], volume annexe, Tōkyō, Daitō shuppansha.

ORZECH, Charles D.

1998 Politics and Transcendent Wisdom: The Scripture for Humane Kings in the Creation of Chinese Buddhism, University Park (Pennsylvania), The Pennsylvania State University Press.

PELLIOT, Paul

1911 Le rôle des apocryphes bouddhiques en Asie centrale et en Chine, manuscrit inédit, discours prononcé à l'Académie des inscriptions et belles-lettres, [C.R. des séances, 1911 , p. 290-291 et Journal asiatique, I (1911), p. 579580].

SAKANO Eihan 圾野榮範

1976 Kongōchōgyō ni kansuru kenkyū 金剛頂經に關する研究 [Étude concernant le Vajraśekhara-sūtra], Tōkyō, Kokusho kankōkai.

SEIDEL, Anna

1990 «Chronicle of Taoist Studies in the West 1950-1990», Cahiers d'ExtrêmeAsie, 5 (1989-90), p. 223-347.

SHIRASU Jōshin 白須淨真

1974 «Donkō ni okeru "Ekkōrinkyō" no denshō - Toruban shihaiki no chūgen miden kanyaku kyōten no kenkyū -» 敦煌における 迴向輪經の 傳承 - 吖蕃支配期の中原卡傳漢譯經典の研究 [Transmission à Dunhuang du Sütra traitant du transfert [des mérites] - Étude concernant les sūtra traduits en chinois mais non transmis en Chine centrale durant la période d'occupation tibétaine], Bukkyō shigaku 佛教史學 [Études d'histoire du bouddhisme], 17/1, p. 34-69. 
SILK, Jonathan A.

1989 «A Note on the Opening of Buddhist Sūtras », Journal of the Asiatic Society of Bengal, 12/1, p. 158-216.

STEIN, R. A.

1971 «Illumination subite ou saisie simultanée, note sur la terminologie chinoise et tibétaine », Revue de l'Histoire des Religions, 179, p. 3-30.

1984 "Quelques découvertes récentes dans les manuscrits tibétains", in Les peintures murales et les manuscrits de Dunhuang, Colloque franco-chinois, Paris, Éditions de la Fondation Singer-Polignac, p. 21-24.

STRICKMANN, Michel

1982 "India in the Chinese Looking-Glass», in The Silk-Route and the Diamond Path, D. E. Klimburg-Salter éd., Los Angeles, University of California Art Council, p. 52-63.

1990 «The Consecration Sütra: A Buddhist Book of Spells », in Chinese Buddhist Apocrypha, Robert J. Buswell éd., Honolulu, University of Hawai'i Press, p. $75-118$.

TAKAKUSU, J.

1896 A Record of the Buddhist Religion as Practised in India and the Malay Archipelao (A.D. 671-695) by I-tsing, translated, Oxford, Clarendon Press (many Indian reprints).

TANAKA Kimiaki 世中公明

2000 «Kongōshunkyō to Chibetto bukkyō» 金剛峻經とチベット佛教 [Jingangjun jing et le bouddhisme tibétain], Gigi butten no sōkateki kenky $\bar{u}$ 疑临佛典 0 綜合的研究 [Recherches concernant les apocryphes bouddhiques], sous la dir. de Kimura Kiyotaka, Tōkyō, Université de Tōkyō, p. $8-24$

TANAKA Ryōsho 用中良昭

1974-77 «Gisaku no mikkyō bunken ni arawareta Zenshū sōtōsetsu - Tonkō shutsudo Periobon 3913 gō no shōkai» 疑作 の密教文獻に現 われた禪宗祖統說－敦煌出土ペリオ 本 3913 號の介紹 [Le lignage patriarcal du Chan selon un document apocryphe tantrique - Présentation du manuscrit de Dunhuang, P. 3913], I et II, Shūkyōgaku ronshī 宗 教學論集 [Mélanges sur l'études des religions], 7 (1974), p. 93-115 et 8 (1977), p. 297-315.

TEISER, Stephen F.

1988 The Gost Festival in Medieval China, Princeton, Princeton University Press.

1994 The Scripture on the Ten Kings and the Making of Purgatory in Medieval Chinese Buddhism, Honolulu, University of Hawai'i Press.

TOKUNO Kyoko

1990 "The Evaluation of Indigenous Scriptures in Chinese Buddhist Bibliographical Catalogues », in Chinese Buddhist Apocrypha, Robert J. Buswell éd., Honolulu, University of Hawai'i Press, p. 31-74.

TOLA, Fernando \& DRAGONETTI, Carmen 1999 « Ekam Samayam », Indo-Iranian Journal, 42, p. 53-55.

UEYAMA Daishun 上山大峻

1967-68 «Daibangoku daitoku sanzō hōshi shamon Hōsei no kenkyū » 大蕃國大德三藏法師沙門法成 の研究 (A Study of the Life and Works of 
Fa-ch'eng (Hgo Chos grub), a Translator of the Buddhist Texts at Tunhuang under Tibetan Rule), I et II, Tōhō gakuhō 東方學報 (Journal of Oriental Studies), (Kyōto), 38, p. 133-198 et 39, p. 119-222.

1972 «Donsen yaku "Kongōdan kōdai seijō dalanikyō" - haseiki Ansei ni okeru

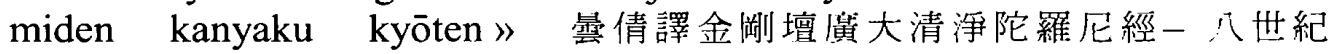
安西におけ る未傳經典 - [Le Jingantan guangda qingjing tuoluoni jing - sūtra traduit en chinois par Tanqing du VIII ${ }^{\mathrm{e}}$ siècle à Anxi, non transmis dans la Chine centrale], Ryūkoku daigaku ronshō 龍谷大學論集 (The Journal of Ryukoku University), 399, p. 60-82.

1981 «Étude des manuscrits tibétains de Dunhuang relatifs au bouddhisme de dhyāna », Journal asiatique, 269/1-2, p. 287-295.

WANG Chongmin 王重民

1983 Dunhuang yishu zongmu suoyin 敦煌遺書總日索引 [Catalogue général et index des manuscrits de Dunhuang], Beijing, Zhonghua shuju [d'après la $1^{\text {re }}$ éd. 1962].

WANG-TOUTAIN, Françoise

1998 Le bodhisattva Ksitigarbha en Chine du $V^{e}$ au XIIF siècle, Paris, EFEO (Monographies, 185).

YABUKI Keiki 矢吹慶輝

1927 Sankaikyō no kenkyū 三階教之研究 [Étude sur la secte des Trois Degrés], Tōkyō, Iwanami shoten.

1933 Meisha yoin kaisetsu 鳴沙餘韻解說 [Interprétation des quelques manuscrits de Dunhuang], Tōkyō, Iwanami shoten.

YORITOMI Motohiro 賴富本宏

1984 «Chūgoku mikkyōshi ni okeru Tonkō bunken»中國密教史 におけ る 敦煌文獻 [Les documents de Dunhuang dans l'histoire du tantrisme chinois], in Kōza Tonkō: Tonkō to Chūgoku bukkyō 講座敦煌：敦煌 と中國佛教 [Leçons sur Dunhuang：Dunhuang et le bouddhisme chinois], Makita Tairyō 牧用謗亮 et Fukui Bumimasa 福井文雅 éd., Tōkyō, Daitō shuppansha, p. 155-166.

YUYAMA Akira

2000 Eugène Burnouf. The Background to his Research into the Lotus Sutra, The International Research Institute for Advanced Buddhology, Tōkyō, Soka University.

ZÜRCHER, Erik

1959 The Buddhist Conquest of China. The Spread and Adaptation of Buddhism in Early Medieval China, Leiden, E. J. Brill, 1959.

1980 "Buddhist Influence on Early Taoism - A Survey of Scripture Evidence », T'oung Pao, 66/1-3, p. 84-147.

1982 " "Prince Moonlight", Messianism and Eschatology in Early Medieval Chinese Buddhism », T'oung Pao, 68/1-3, p. 1-75.

1991 «A New Look at the Earliest Chinese Buddhist Texts », in From Benares to Beijing. Essays on Buddhism and Chinese Religion in Honour of Prof. Jan Yün-hua, Koichi Shinohara et Gregory Schopen éd., Oakville / New York / London, Mosaic Press, p. 277-304. 